\title{
VARIATION OF LOG CANONICAL THRESHOLDS IN LINEAR SYSTEMS
}

\author{
FLORIN AMBRO
}

\author{
Dedicated to the memory of Professor Şerban Basarab
}

\begin{abstract}
We investigate the variation of log canonical thresholds in (graded) linear systems. For toric log Fano varieties, we give a sharp lower bound for log canonical thresholds of the anticanonical members in terms of the global minimal log discrepancy.
\end{abstract}

\section{INTRODUCTION}

An interesting question in the classification theory of algebraic varieties is the conjecture of Alexeev and Borisov brothers: (singular) Fano varieties $X$ of fixed dimension $d$ and with minimal $\log$ discrepancy $\operatorname{mld}(X) \geq \epsilon>0$, belong to a bounded family. This conjecture is known if $d=2$ [1] or $X$ is a toric variety [7]. To a Fano variety $X$ we associate the $\alpha$-invariant

$$
\gamma(X)=\inf \left\{\operatorname{lct}\left(X ; \frac{D_{n}}{n}\right) ; n \geq 1, D_{n} \in\left|-n K_{X}\right|\right\} .
$$

It is known that $\gamma(X) \cdot \sqrt[d]{\left(-K_{X}\right)^{d}} \leq d$, so the anticanonical volume is bounded above if the $\alpha$ invariant is bounded away from zero. It follows that the above conjecture reduces to two local statements: a) a lower bound $\gamma(X) \geq \gamma(d, \epsilon)>0$, and b) an upper bound $r \leq r(d, \epsilon)$ for the smallest integer $r \geq 1$ such that $r K_{X}$ is Cartier. The first aim of this paper is to propose a sharp lower bound $\gamma(d, \epsilon)$, and to establish it in the toric case.

Theorem 0.1. Let $(X, B)$ be a toric log Fano variety, with $\operatorname{dim} X=d$ and $\operatorname{mld}(X, B) \geq \frac{1}{q}$, for some integer $q \geq 1$. Then

$$
\gamma(X, B)=\inf \left\{\operatorname{lct}\left(X, B ; \frac{D_{n}}{n}\right) ; n \geq 1, D_{n} \in\left|-n K_{X}-n B\right|\right\} \geq \frac{q}{u_{d+1, q}},
$$

where $\left(u_{p, q}\right)_{p, q \geq 1}$ is the sequence of integers defined recursively by $u_{1, q}=q, u_{p+1, q}=u_{p, q}\left(1+u_{p, q}\right)$.

Theorem 0.1 is sharp (see Example 5.3). An interesting feature is that the type of coefficients of $B$ do not matter. We expect the same bound holds in the non-toric case. This is easy to see in dimension one, but unclear in dimension two.

The $\alpha$-invariant can be localized, and defined for polarized log varieties $(X, B ; H)$. One defines $\gamma(X, B ; H)=\inf _{P \in X} n \cdot \gamma_{P}(X, B ;|n H|)$, where $\gamma_{P}(X, B ;|n H|)=\inf \left\{\operatorname{lct}_{P}(X, B ; D) ; D \in|-n H|\right\}$. The second aim of the paper is to study the variation of $\operatorname{lct}_{P}(X, B ; D)$ in the variables $P, D$, as suggested in [2]. The variation in $D$ is implicit in the work of Viehweg [17, Sections 5.3,8.2]. He considers only the special case when $B=0$ and $X$ has klt singularities, and replaces $\gamma(X, 0 ; D)$ by $e(X, 0 ; D)$, the largest positive integer $e$ such that $\frac{1}{e}<\operatorname{lct}(X, 0 ; D)$. We extend Viehweg's results to our setting. Allowing non-zero boundaries strengthens some statements, and gives new ones. The main new statement is

\footnotetext{
${ }^{1}$ This work was supported by a grant of the Romanian National Authority for Scientific Research, CNCS UEFISCDI, project number PN-II-RU-TE-2011-3-0097.

${ }^{2} 2010$ Mathematics Subject Classification. Primary: 14B05. Secondary: 14J45.

${ }^{3}$ Keywords: $\log$ variety, $\log$ canonical threshold.
} 
Theorem 0.2. Let $\pi:(X, B) \rightarrow S$ be a family of log varieties. Then the locus of points $x \in X$ where $\left(X_{\pi(x)}, B_{\pi(x)}\right)$ has log canonical singularities is open.

It follows that log canonical thresholds are lower semi-continuous in flat families. In particular, $\operatorname{lct}_{P}(X, B ; D)$ is lower semicontinuous in two variables $(P, D) \in X \times|n H|$. By projection onto the second factor, we obtain extensions to the log category of the semi-continuity results of Varchenko [16] and Demailly-Kollár [9]. By projection onto the first factor, we obtain that $P \mapsto \gamma_{P}(X, B ;|n H|)$ is lower semi-continuous and takes only finitely many values. It would be interesting to find out if the same holds for the asymptotic version $P \mapsto \gamma_{P}(X, B ; H)$.

We outline the structure of this paper. In Section 1 we introduce families of log varieties. The main result is the openness of the locus where the fibers have log canonical singularities (Theorem 1.8). It follows that $\log$ canonical thresholds are semi-continuous in flat families (Corollary 1.10).

In Section 2, we study the variation of $\log$ canonical thresholds $\operatorname{lct}_{P}(X, B ; D)$ in $P$ and $D$, where $D$ moves in a linear system. The results follow from those of Section 1 applied to the universal divisor of the linear system. We also generalize the product theorem of Viehweg to distinct factors (Theorem 2.7). To an $\mathbb{N}$-graded convex family of linear systems $\Lambda_{\bullet}$ on a given log variety $(X, B)$, we associate the local $\alpha$-invariant at $x$ as follows:

$$
\gamma_{x}\left(X, B ; \Lambda_{\bullet}\right)=\inf \left\{\operatorname{lct}_{x}\left(X, B ; \frac{D_{n}}{n}\right) ; n \geq 1, D_{n} \in \Lambda_{n}\right\}
$$

It is very interesting to study the variation in $x$ of this functional (see Question 2.8), but we can only say little in general. We compute this invariant if $X$ is a curve, recall some known results, and express it in terms of width. We can say more in the toric case. In Section 3, we compute the local $\alpha$-invariant in the generic point of the invariant primes of a toric variety, and show that their minimum is exactly the global $\alpha$-invariant (Theorem 3.4). In particular, we can compute combinatorially the $\alpha$-invariant of a line bundle on a toric variety. With some extra assumptions, this was independently obtained in [8, Theorem 3.4], with analytic methods.

The main result of Section 4 is Theorem 4.6, a logarithmic effective version of a diophantine approximation result of Hensley [11, Lemma 2]. The special case $q=1$ was solved by Averkov [5, Theorem 2.1], and our proof is inspired from his. We also give sharp versions of the original results of Hensley [11.

In Section 5, we give the sharp lower bound for the $\alpha$-invariant of a toric log Fano (Theorem 5.1 ), a result essentially equivalent to Theorem 4.6. In particular, we obtain an upper bound for the anti log canonical volume (Corollary 5.2). Theorem 5.4 extends to the log category and simplifies the proof of the finiteness of $d$-dimensional $\epsilon$-log canonical toric Fano varieties [7]. We end Section 5 with the simplest examples of toric log Fano varieties, where we can explicitly compute both the minimal log discrepancy and the $\alpha$-invariant, and see their relation with diophantine approximation.

\section{FAMILIES OF LOG VARIETIES}

Relative effective Cartier divisors. Throughout this paper, we fix a base field $k$, algebraically closed and of characteristic zero. By scheme we mean a $k$-scheme of finite type.

Let $\pi: X \rightarrow S$ be a flat morphism of schemes, and $D$ an effective Cartier divisor on $X$. Recall that $D$ is called relative over $S$ if one of the following equivalent conditions holds:

a) $D$ is flat over $S$.

b) for every point $x \in X$ and a local equation $f$ for $D$ at $x, f$ does not divide zero in $\mathcal{O}_{X_{\pi(x)}, x}$.

c) for every $s \in S$, Supp $D$ contains no associated prime of $X_{s}$.

If the fibers of $\pi$ are reduced, c) means that $\operatorname{Supp} D$ contains no irreducible component of a fiber of $\pi$. If $S^{\prime} \rightarrow S$ is a morphism of schemes, the pullback of $D$ is a well defined effective Cartier divisor on $X \times{ }_{S} S^{\prime}$, relative over $S^{\prime}$. 
Let $\pi: X \rightarrow S$ be a flat morphism of schemes. An effective $\mathbb{Q}$-Cartier divisor on $X$ relative over $S$ is a formal product $x D$, where $x \geq 0$ is a rational number and $D$ is an effective Cartier divisor on $X$ relative over $S$. If $r x \in \mathbb{Z}$, we can write it as $\frac{1}{r} D^{\prime}$, where $D^{\prime}=r x D$ is an effective Cartier divisor on $X$ relative over $S$.

We will use the following special case of [10, Proposition 3.5]:

Lemma 1.1. Let $f: X \rightarrow S$ be a flat morphism of schemes, whose fibers satisfy Serre's property $\left(S_{2}\right)$. Let $w: U \subseteq X$ be an open subset such that its complement $Z$ satisfies $\operatorname{codim}\left(Z_{s}, X_{s}\right) \geq 2$ for every $s \in S(k)$. Let $\mathcal{L}$ be an invertible $\mathcal{O}_{X}$-module. Then $\mathcal{H}_{Z}^{i}(\mathcal{L})=0(i=0,1)$, that is $\mathcal{L} \stackrel{\sim}{\rightarrow} w_{*}\left(\left.\mathcal{L}\right|_{U}\right)$.

Log varieties, log canonical thresholds. Let $(X / k, B)$ be a log variety. Log discrepancies in geometric valuations of $X$, and minimal $\log$ discrepancies in scheme points, or closed subsets of $X$, are well defined (see for example [2, 4]). Denote by $(X, B)_{l c}$ the largest open locus in $X$ where $(X, B)$ has $\log$ canonical singularities, and $(X, B)_{-\infty}$ its complement. For an effective $\mathbb{R}$-divisor $D$ on $X$ and a scheme point $x \in(X, B)_{l c}$, the log canonical threshold at $x$ of $D$ with respect to $(X, B)$ is defined as

$$
\operatorname{lct}_{x}(X, B ; D)=\sup \left\{t \geq 0 ; \operatorname{mld}_{x}(X, B+t D) \geq 0\right\} .
$$

It is $+\infty$ if $x \notin \operatorname{Supp} D, 0$ if $x \in \operatorname{Supp} D$ and $\operatorname{mld}_{x}(X, B)=0$, and a positive real number if $x \in \operatorname{Supp} D$ and $\operatorname{mld}_{x}(X, B)>0$. It is rational if so are $B, D$ near $x$. The reciprocal

$$
\mu_{x}(X, B ; D)=1 / \operatorname{lct}_{x}(X, B ; D)
$$

is called the Arnold multiplicity at $x$ of $D$ with respect to $(X, B)$. It is 0 if $x \notin \operatorname{Supp} D$, $+\infty$ if $x \in \operatorname{Supp} D$ and $\operatorname{mld}_{x}(X, B)=0$, and a positive real number if $x \in \operatorname{Supp} D$ and $\operatorname{mld}_{x}(X, B)>0$.

Families of $\log$ varieties. A family of $\log$ varieties $(X / S, B)$ consists of the following data:

a) a flat morphism of schemes $\pi: X \rightarrow S$, such that $X_{s}$ is normal for every $s \in S$;

b) an effective $\mathbb{Q}$-Cartier divisor $B$ defined on $U_{\pi}$ and relative over $S$, where $w: U_{\pi} \subseteq X$ is the open locus where the morphism $\pi$ is smooth,

satisfying the following property: there exists an integer $r \geq 1$ such that $r B$ is Cartier and the $\mathcal{O}_{X}$-module $w_{*}\left(\left(\Omega_{U / S}^{t o p}\right)^{\otimes r}(r B)\right)$ is locally free. The smallest $r$ with this property is called the index of the family.

Here $\Omega_{U / S}^{t o p}$ is the top exterior product of $\Omega_{X / S}^{1}$, corresponding to the locally constant dimension of the fibers. Property a) implies that $Z=X \backslash U$ is the union of the singular locus of $X_{s}$, after all $s \in S(k)$. In particular, $\operatorname{codim}\left(Z_{s}, X_{s}\right) \geq 2$ for every $s \in S(k)$. Recall that normality of a scheme is defined locally, so it does not imply irreducibility. The fibers of $\pi$ are normal if and only if $X_{s}$ is normal for every $s \in S(k)$. Even if the latter are irreducible, some fibers over non-closed points may be reducible.

Consider a family of $\log$ varieties of index $r$. For $l \in r \mathbb{Z}, l B$ is a Cartier divisor on $U$, and we can define

$$
\omega^{[l]}=w_{*}\left(\left(\Omega_{U / S}^{t o p}\right)^{\otimes l} \otimes \mathcal{O}_{U}(l B)\right) .
$$

The $\mathcal{O}_{X^{-}}$module $\omega^{[l]}$ is coherent (EGA IV, Proposition 5.11.1). By assumption, $\omega^{[r]}$ is an invertible $\mathcal{O}_{X}$-module.

Lemma 1.2. $\left(\omega^{[r]}\right)^{\otimes \frac{l}{r}} \stackrel{\sim}{\rightarrow} \omega^{[l]}$ for every $l \in r \mathbb{Z}$.

Proof. We have natural multiplication maps $\omega^{[l]} \otimes \omega^{\left[l^{\prime}\right]} \rightarrow \omega^{\left[l+l^{\prime}\right]}$. By Lemma $1.1, \omega^{[0]}=\mathcal{O}_{X}$. Therefore suffices to show that $\omega^{[r]} \otimes \omega^{[l]} \rightarrow \omega^{[r+l]}$ is an isomorphism. Indeed, denote $\mathcal{F}=\omega^{[r]} \otimes \omega^{[l]}$. Our homomorphism factors as

$$
\mathcal{F} \rightarrow w_{*}\left(\left.\mathcal{F}\right|_{U}\right) \stackrel{\sim}{\rightarrow} \omega^{[r+l]}
$$


We have $\mathcal{H}_{Z}^{i}(\mathcal{F})=0(i=0,1)$, since $\omega^{[l]}$ satisfies this property by Lemma 1.1, and $\omega^{[r]}$ is locally trivial. Therefore the first map is also an isomorphism.

Lemma 1.3. Let $(X / S, B)$ be a family of log varieties of index $r$. Let $g: S^{\prime} \rightarrow S$ be a morphism of schemes. Consider the induced base change diagram

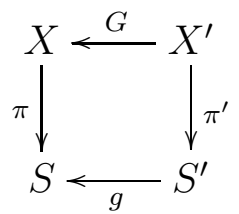

Then $U_{\pi^{\prime}}=G^{-1}\left(U_{\pi}\right),\left(X^{\prime} / S^{\prime},\left(\left.G\right|_{U_{\pi^{\prime}}}\right)^{*} B\right)$ is a family of log varieties over $S^{\prime}$, and we have natural isomorphisms

$$
G^{*}\left(\omega^{[l]}\right) \stackrel{\sim}{\rightarrow} \omega^{[l]}(l \in r \mathbb{Z}) .
$$

Proof. We have $G^{*}\left(\Omega_{X / S}^{1}\right) \stackrel{\sim}{\rightarrow} \Omega_{X^{\prime} / S^{\prime}}^{1}$. Therefore $U_{\pi^{\prime}}=G^{-1}\left(U_{\pi}\right)$ and we have a base change diagram

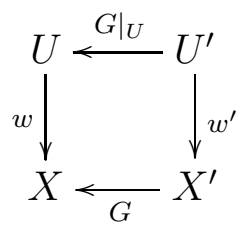

For $l \in r \mathbb{Z}$, we obtain a natural homomorphism

$$
\left.\left.\left.G^{*}\left(\omega^{[l]}\right)=G^{*} w_{*}\left(\left(\Omega_{U / S}^{t o p}\right)^{\otimes l}(l B)\right) \rightarrow w_{*}^{\prime}\left(\left(\left.G\right|_{U}\right)^{*}\left(\Omega_{U / S}^{t o p}\right)^{\otimes l}(l B)\right)\right) \stackrel{\sim}{\rightarrow} w_{*}^{\prime}\left(\Omega_{U^{\prime} / S^{\prime}}^{t o p}\right)^{\otimes l}\left(l B^{\prime}\right)\right)\right)=\omega^{[l[l]} .
$$

If we denote this homomorphism by $\mathcal{F}^{\prime} \rightarrow \mathcal{G}^{\prime}$, it factors as $\mathcal{F}^{\prime} \rightarrow w_{*}^{\prime}\left(\left.\mathcal{F}^{\prime}\right|_{U^{\prime}}\right) \stackrel{\sim}{\rightarrow} \mathcal{G}^{\prime}$. Since $\omega^{[l]}$ is locally trivial, so is $\mathcal{F}^{\prime}$. By Lemma 1.1, $\mathcal{H}_{Z^{\prime}}^{i}\left(\mathcal{F}^{\prime}\right)=0(i=0,1)$. Therefore $\mathcal{F}^{\prime} \rightarrow w_{*}^{\prime}\left(\left.\mathcal{F}^{\prime}\right|_{U^{\prime}}\right)$ is also an isomorphism.

In particular, for every $s \in S(k)$, the fiber $X_{s}$ is a normal variety, $r B_{s}$ is an effective Cartier divisor on $U_{s}=X_{s} \backslash \operatorname{Sing}\left(X_{s}\right)$, and if $r \bar{B}_{s}$ is the effective Weil divisor which is the closure of $r B_{s}$ in $X_{s}$, we have a base change diagram

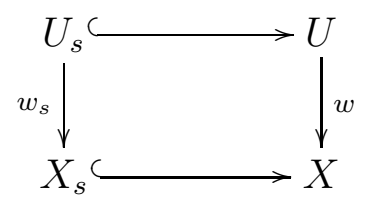

and an isomorphism

$$
\left.\omega^{[r]}\right|_{X_{s}} \stackrel{\sim}{\rightarrow} w_{s *}\left(\left(\Omega_{U_{s} / k}^{t o p}\right){ }^{\otimes r}\left(r B_{s}\right)\right)=\mathcal{O}_{X_{s}}\left(r K_{X_{s}}+r \bar{B}_{s}\right) .
$$

Therefore $r K_{X_{s}}+r \bar{B}_{s}$ is Cartier, so that $\left(X_{s}, \bar{B}_{s}\right)$ is a log variety. We think of $\left(X_{s}, \bar{B}_{s}\right)(s \in S(k))$ as an algebraic family of $\log$ varieties parametrized by $S$. The boundary coefficients may vary. To simplify notation, we denote $\bar{B}_{s}$ by $B_{s}$.

Examples 1.4. $\quad 1)$ Let $\pi: X \rightarrow S$ be a smooth morphism of schemes. Then $(X / S, B)$ is a family of $\log$ varieties over $S$ if and only if $B=\frac{1}{r} D$, for some integer $r \geq 1$ and an effective Cartier divisor $D$ on $X$ which is relative over $S$. The fibers are the log varieties $\left(X_{s},\left.\frac{1}{r} D\right|_{X_{s}}\right)(s \in S(k))$.

2) Let $\left(F, B_{F}\right)$ be a log variety over $k$. Let $S$ be a scheme. Denote by $B_{F} \times S$ the $\mathbb{Q}$ Cartier divisor $p_{1}^{*}\left(B_{F^{0}}\right)$, defined on $F^{0} \times S$, where $F^{0}$ is the smooth locus of $F$. Then $\left(F \times S / S, B_{F} \times S\right)$ is the trivial family of $\log$ varieties over $S$, with constant fiber $\left(F, B_{F}\right)$. 
3) Let $(X / S, B)$ be a family of log varieties. Let $D$ be an effective Cartier divisor on $X$, relative over $S$. Then $(X / S, B+t D)$ is a family of $\log$ divisors for every rational $t \geq 0$.

4) A family of $\log$ curves consists of a smooth morphism $\pi: X \rightarrow S$ of relative dimension one, endowed with an effective $\mathbb{Q}$-Cartier divisor $B$, relative over $S$. If $r$ is the index of the family, then $r B=D$ is an effective Cartier divisor on $X$ which is finite flat over $S$. The $\mathcal{O}_{S}$-module $\pi_{*} \mathcal{O}_{D}$ is locally free.

Lemma 1.5. Let $\pi: X \rightarrow S$ be a flat morphism of schemes with normal fibers, and $S$ smooth over $k$. Then $(X / S, B)$ is a family of log varieties if and only if $(X / k, B)$ is a log variety and the boundary supports no irreducible components of fibers of $\pi$. Moreover, they have the same index $r$, and $\pi^{*} \omega_{S / k}^{\otimes r} \otimes \omega_{(X / S, B)}^{[r]} \simeq \omega_{(X / k, B)}^{[r]}$.

Proof. Since $S$ and the fibers are normal, so is $X$. Let $U=U_{\pi}$ be the smooth locus of $\pi$. It follows that $\operatorname{codim}(X \backslash U, X) \geq 2$. In particular, $l B$ is Cartier on $U$ if and only if $l \bar{B}$ is a Weil divisor on $X$. We have a short exact sequence

$$
0 \rightarrow \pi^{*} \Omega_{S / k}^{1} \rightarrow \Omega_{U / k}^{1} \rightarrow \Omega_{U / S}^{1} \rightarrow 0 .
$$

It induces an isomorphism $\pi^{*} \Omega_{S / k}^{t o p} \otimes \Omega_{U / S}^{t o p} \simeq \Omega_{U / k}^{t o p}$. If $l B$ is Cartier on $U$, we obtain an isomorphism

$$
\pi^{*} \omega_{S / k}^{\otimes l} \otimes \omega_{(X / S, B)}^{[l]} \simeq \omega_{(X / k, B)}^{[l]} .
$$

Therefore $\omega_{(X / S, B)}^{[l]}$ is locally free if and only if $\omega_{(X / k, B)}^{[l]}$ is locally free, and the first claim follows. The second follows from the first.

Lemma 1.6. Let $(X, B)$ be a log variety. Let $f: X \rightarrow S$ be a morphism, with $S$ a reduced scheme. Then there exists an open subset $\emptyset \neq V \subseteq S$ such that $\left.(X, B)\right|_{f^{-1} V} \rightarrow V$ is a family of log varieties.

Proof. We may shrink $S$ to an open subset, so that $S$ is smooth, and $f$ is flat with normal fibers. Let $U \subseteq X$ be the smooth locus of $f$. Let $r \geq 1$ such that $r K_{X}+r B$ is Cartier. We may further shrink $S$ so that the effective Cartier divisor $\left.r B\right|_{U}$ becomes flat over $S$. By Lemma 1.5, $\left(X / S,\left.B\right|_{U}\right)$ is a family of $\log$ varieties.

Lemma 1.7. Let $\pi:(X, B) \rightarrow S$ be a family of log varieties. Let $S$ be regular at $s$, and choose a regular system of parameters $\left(h_{i}\right)_{i}$ for $\mathcal{O}_{S, s}$. The following are equivalent for $x \in X_{s}$ :

a) the fiber $\left(X_{s}, B_{s}\right)$ has log canonical singularities near $x$;

b) the log variety $\left(X, B+\pi^{*} \Sigma_{s}\right)$ has log canonical singularities near $x$, where $\Sigma_{s}=\sum_{i} \operatorname{div}\left(h_{i}\right)$.

Proof. Let $S^{\prime}=\operatorname{div}\left(h_{j}\right)$. It is defined locally near $s$, but we may shrink $S$ to a neighborhood of $s$. Let $X^{\prime}=\pi^{*}\left(S^{\prime}\right), \pi^{\prime}: X^{\prime} \rightarrow S^{\prime}$ the induced morphism, and $B^{\prime}=\left.B\right|_{U_{\pi^{\prime}}}$. The base change data $\left(X^{\prime}, B^{\prime}\right) \rightarrow S^{\prime} \ni s,\left(\left.h_{i}\right|_{S^{\prime}}\right)_{i \neq j}$ satisfy the same properties. Now $\left(X, B+X^{\prime}\right)$ is a log variety with lc center $X^{\prime}$, a normal Cartier divisor in $X$. Therefore the different is zero, and the codimension one adjunction formula is

$$
\left.\left(K_{X}+B+X^{\prime}\right)\right|_{X^{\prime}}=K_{X^{\prime}}+B^{\prime} .
$$

If we denote $\Sigma_{s}^{\prime}=\sum_{i \neq j} \operatorname{div}\left(h_{i}\right)$, we obtain $\left.\left(K_{X}+B+\pi^{*} \Sigma_{s}\right)\right|_{X^{\prime}}=K_{X^{\prime}}+B^{\prime}+\pi^{\prime *} \Sigma_{s}^{\prime}$. By Inversion of Adjunction [12], $\left(X, B+\pi^{*} \Sigma_{s}\right)$ has log canonical singularities near $x$ if and only if $\left(X^{\prime}, B^{\prime}+\pi^{\prime *} \Sigma_{s}^{\prime}\right)$ has $\log$ canonical singularities near $x$. Iterating this argument proves the equivalence.

Theorem 1.8. Let $\pi:(X, B) \rightarrow S$ be a family of log varieties. Then the locus of points $x \in X$ where $\left(X_{\pi(x)}, B_{\pi(x)}\right)$ has log canonical singularities is open. 
Proof. We have to show that the complement $Z(\pi)=\cup_{s \in S(k)}\left(X_{s}, B_{s}\right)_{-\infty}$ is closed in $X$. For this, it suffices to construct a non-empty open subset $V \subseteq S$ such that $Z(\pi) \mid \pi^{-1}(V)$ is closed. Indeed, replacing $S$ by $S^{\prime}=S \backslash V$ and $\pi$ by the induced by base change family, we have

$$
Z(\pi)=\left(Z(\pi) \mid \pi^{-1}(V)\right) \cup Z\left(\pi \mid S^{\prime}\right) .
$$

By noetherian induction, $Z\left(\pi \mid S^{\prime}\right)$ is closed. Therefore $Z(\pi)$ is closed.

It remains to prove the claim. For this, we may base change with the reduced structure on $S$ and then restrict to the regular locus. Therefore $S$ is regular. By Lemma 1.5, $(X, B)$ is a log variety. We show that we have an inclusion $(X, B)_{-\infty} \subseteq Z(\pi)$, which is an equality over some non-empty open subset of $S$.

For the inclusion, let $x \in X \backslash Z(\pi)$, so that $\left(X_{\pi(x)}, B_{\pi(x)}\right)$ has log canonical singularities at $x$. By Lemma 1.7, $\left(X, B+\pi^{*} \Sigma_{s}\right)$ has $\log$ canonical singularities at $x$. Therefore $(X, B)$ has $\log$ canonical singularities at $x$, that is $x \notin(X, B)_{-\infty}$.

By Hironaka, there exists a desingularization $\mu: X^{\prime} \rightarrow X$ and a normal crossing divisor $\sum_{i} E_{i}$ which supports $B^{\prime}=\mu^{*}\left(K_{X}+B\right)-K_{X^{\prime}}$. After shrinking $S$ to an open subset, the morphism $\left(X^{\prime}, \sum_{i} E_{i}\right) \rightarrow S$ becomes log smooth. In this case, we show that the inclusion is an equality. Assuming $(X, B)$ has log canonical singularities at $x$, we have to show that $\left(X_{s}, B_{s}\right)$ has $\log$ canonical singularities at $x$, where $s=\pi(x)$. We may shrink $X$ to a neighborhood of $x$, and suppose $(X, B)$ has $\log$ canonical singularities. That is the coefficients of $B^{\prime}$ are at most 1 . By adjunction, we have

$$
\mu_{s}^{*}\left(K_{X_{s}}+B_{s}\right)=K_{X_{s}^{\prime}}+B_{s}^{\prime}
$$

where $B_{s}^{\prime}=\left.\sum_{i} b_{i} E_{i}\right|_{X_{s}^{\prime}}$. Since $\left(X^{\prime}, \sum_{i} E_{i}\right) \rightarrow S$ is log smooth, a prime divisor on $X_{s}^{\prime}$ is contained in at most one $E_{i}$. Therefore the coefficients of $B_{s}^{\prime}$ are some of the $b_{i}$ 's, so at most 1 . Therefore $\left(X_{s}, B_{s}\right)$ has log canonical singularities at $x$.

Proposition 1.9. Let $\pi:(X, B) \rightarrow S$ be a real family of log varieties whose fibers have at most log canonical singularities. Let $D$ be an effective Cartier divisor on $X$, relative over $S$. Then the function $X \ni x \mapsto \operatorname{lct}_{x}\left(X_{\pi(x)}, B_{\pi(x)} ; D_{\pi(x)}\right)$ is lower semi-continuous and takes only finitely many values.

Proof. Fix $t \geq 0$. Then $\operatorname{lct}_{x}\left(X_{\pi(x)}, B_{\pi(x)} ; D_{\pi(x)}\right) \geq t$ if and only if $\left(X_{\pi(x)}, B_{\pi(x)}+t D_{\pi(x)}\right)$ has $\log$ canonical singularities at $x$. Since $\left(X, B+\left.t D\right|_{U}\right) \rightarrow S$ is a family of log varieties, the locus of such $x$ is open by Theorem 1.8. Therefore the function is lower semi-continuous.

To show that the function takes only finitely many values, it suffices to prove this holds over some open non-empty subset of $S$ (by noetherian induction). Then we may assume $S$ is reduced and regular. Let $X^{\prime} \rightarrow X$ be a desingularization, endowed with a normal crossing divisor $\sum_{i} E_{i}$ which supports both $B^{\prime}=\mu^{*}\left(K_{X}+B\right)-K_{X^{\prime}}$ and $D^{\prime}=\mu^{*} D$. We finally shrink $S$ to an open subset such that $\left(X, \sum_{i} E_{i}\right) \rightarrow S$ becomes log smooth. We have

$$
\mu_{s}^{*}\left(K_{X_{s}}+B_{s}+t D_{s}\right)=K_{X_{s}^{\prime}}+B_{s}^{\prime}+t D_{s}^{\prime},
$$

and the coefficients of $B_{s}^{\prime}+t D_{s}^{\prime}$ are some of the $b_{i}^{\prime}+t d_{i}^{\prime}$. Therefore each $\operatorname{lct}_{x}\left(X_{s}, B_{s} ; D_{s}\right)$ is either $+\infty$, or the largest $t \geq 0$ such that $b_{i}^{\prime}+t d_{i}^{\prime} \leq 1$ for certain $i$. They belong to a finite set.

Corollary 1.10. Let $\pi:(X, B) \rightarrow S$ be a real family of log varieties. Let $Z \subseteq X$ be a closed subset, such that $Z \rightarrow S$ is proper surjective, and $\left(X_{s}, B_{s}\right)$ has $\log$ canonical singularities near $Z_{s}$ for every $s \in S$. Let $D$ be an effective Cartier divisor on $X$, relative over $S$. Then the function $S \ni s \mapsto \operatorname{lct}_{Z_{s}}\left(X_{s}, B_{s} ; D_{s}\right)$ is lower semi-continuous and takes only finitely many values.

Proof. By Theorem 1.8, we may shrink $X$ near $Z$, so that the fibers of $\pi$ have log canonical singularities. Denote $\gamma(x)=\operatorname{lct}_{x}\left(X_{\pi(x)}, B_{\pi(x)} ; D_{\pi(x)}\right)$. Then $\operatorname{lct}_{Z_{s}}\left(X_{s}, B_{s} ; D_{s}\right)=\min _{x \in Z_{s}} \gamma(x)$, and

$$
\left\{s \in S ; \operatorname{lct}_{Z_{s}}\left(X_{s}, B_{s} ; D_{s}\right)<t\right\}=\pi(Z \cap\{x \in X ; \gamma(x)<t\}) .
$$


So it follows from Proposition [1.9.

\section{LCT-VARIATION IN A LINEAR SYSTEM}

Let $(X / k, B)$ be a geometric log variety. Let $\Lambda$ be a non-empty, finite dimensional linear system on $X$. That is $\mathcal{L}$ is an invertible $\mathcal{O}_{X}$-module, $V \subseteq \Gamma(X, \mathcal{L})$ is a non-zero finite dimensional $k$-vector subspace, and $\Lambda$ is the family of divisors of zeros of sections in $V$. Using a basis of $V$, we may identify $\Lambda$ with $\mathbb{P}_{k}^{n}$, where $n$ is the dimension of $\Lambda$. Inside $X \times \Lambda$ we have the universal divisor $H$, given by $\sum_{i=0}^{n} s_{i}(x) \lambda_{i}=0$, where $s_{0}, \ldots, s_{n}$ is basis of $V$ over $k$.

Proposition 2.1. The function $(X, B)_{l c} \times \Lambda \rightarrow[0, \infty],(P, D) \mapsto \operatorname{lct}_{P}(X, B ; D)$ is lower semicontinuous and takes only finitely many values.

Proof. Denote $\mathcal{X}=X \times X \times \Lambda, S=X \times \Lambda$, and $\pi=p_{23}: \mathcal{X} \rightarrow S$ the projection on the last two factors. Let $\sigma$ be the section of $\pi$ which is the diagonal on $X$ and the identity on $\Lambda$. Let $\mathcal{B}=p_{1}^{*} B$ and $\mathcal{H}=p_{13}^{*} H$. Then $\pi:(\mathcal{X}, \mathcal{B}) \rightarrow S$ is a family of $\log$ varieties, $\sigma$ is a section of $\pi$, and $\mathcal{H}$ is an effective Cartier divisor relative over $S$. For $s=(P, D) \in S$, the fiber $\left(\mathcal{X}_{s}, \mathcal{B}_{s}+t \mathcal{H}_{s}\right)$ is isomorphic to $(X, B+t D)$ and $\sigma(s) \in \mathcal{X}_{s}$ corresponds to $P \in X$. If we restrict the family to $(X, B)_{l c} \times \Lambda$, it becomes $\log$ canonical, and $\operatorname{lct}_{\sigma(s)}\left(\mathcal{X}_{s}, \mathcal{B}_{s} ; \mathcal{H}_{s}\right)=\operatorname{lct}_{P}(X, B ; D)$. We conclude by Proposition 1.9.

Example 2.2. $\operatorname{lct}_{P}(X, B ; D)<+\infty$ if and only if $(P, D) \in H$.

Example 2.3. Endow the affine line $\mathbb{A}_{k}^{1}$ with a boundary $B=\sum_{P} b_{P} P$, where $b_{P} \in[0,1]$, and only finitely many are non-zero. Let $f_{0}, \ldots, f_{n} \in k[t]$ be polynomials, linearly independent over $k$. They induce a linear system $\Lambda=\left\{D_{\lambda}=\operatorname{div}\left(\sum_{i} \lambda_{i} f_{i}\right) ; \lambda \in \mathbb{P}^{n}\right\}$. Denote by $\partial$ the canonical derivation of $k[t]$. Then $\operatorname{lct}_{P}\left(\mathbb{A}^{1}, B ; D_{\lambda}\right)<t$ if and only if $\sum_{i} \frac{\partial^{m} f_{i}(P)}{m !} \lambda_{i}=0$ for every integer $1 \leq m \leq \frac{1-b_{P}}{t}$.

Theorem 2.4. Let $x \in(X, B)_{l c}$ be a scheme point. The function $\operatorname{lct}_{x}(X, B ; \cdot): \Lambda \rightarrow[0,+\infty]$ is lower semicontinuous and takes only finitely many values.

Proof. We have $\operatorname{lct}_{x}(X, B ; D)=\max _{P \in \bar{x}} \operatorname{lct}_{P}(X, B ; D)$. By Proposition 2.1, it takes only finitely many values. For $t>0$, we have

$$
\left\{D \in \Lambda ; \operatorname{lct}_{x}(X, B ; D)<t\right\}=\cap_{P \in \bar{x}}\left\{D \in \Lambda ; \operatorname{lct}_{P}(X, B ; D)<t\right\} .
$$

Each term on the right hand side is closed, by Proposition 2.1. Therefore the left hand side is also closed.

In particular, $\operatorname{lct}_{x}(X, B ; \cdot)$ attains its maximum (resp. minimum) on a dense open (resp. special closed) subset of $\Lambda$. Define

$$
\gamma_{x}(X, B ; \Lambda)=\min _{D \in \Lambda} \operatorname{lct}_{x}(X, B ; D) .
$$

Denote $\mu_{x}(X, B ; \Lambda)=1 / \gamma_{x}(X, B ; \Lambda)$, so that $\mu_{x}(X, B ; \Lambda)=\max _{D \in \Lambda} \mu_{x}(X, B ; D)$.

Theorem 2.5. The function $(X, B)_{l c} \rightarrow[0,+\infty], P \mapsto \gamma_{P}(X, B ; \Lambda)$ is lower semicontinuous and takes only finitely many values.

Proof. By Proposition 2.1, the values belong to a finite set. And $\left\{P \in(X, B)_{l c} ; \gamma_{P}(X, B ; \Lambda)<t\right\}$ is the projection on the first factor of $\left\{(P, D) \in(X, B)_{l c} \times \Lambda ; \gamma_{P}(X, B ; D)<t\right\}$. The latter is closed by Proposition 2.1, and since $\Lambda$ is proper over $k$, it follows that our level set is closed.

Theorem 2.6. Let $Z \subseteq X$ be a closed subset such that $Z / k$ is proper and $(X, B)$ has log canonical singularities near $Z$. The function $\Lambda \rightarrow[0, \infty], D \mapsto \operatorname{lct}_{Z}(X, B ; D)$ is lower semicontinuous and takes only finitely many values. 
Proof. Denote $\mathcal{X}=X \times_{k} \Lambda, S=\Lambda$, and $\pi: \mathcal{X} \rightarrow S$ the second projection. Let $\mathcal{B}=p_{1}^{*} B$ and $\mathcal{H} \subset X \times \Lambda$ the universal divisor. Then $\pi:(\mathcal{X}, \mathcal{B}) \rightarrow S$ is a family of log varieties, $\mathcal{H}$ is an effective Cartier divisor relative over $S, \mathcal{Z}=Z \times S$ is a closed subset of $X$ which is proper over $S$. For $s=$ $[D] \in S$, the fiber $\left(\mathcal{X}_{s}, \mathcal{B}_{s}+t \mathcal{H}_{s}\right)$ is isomorphic to $(X, B+t D)$ and $\operatorname{lct}_{\mathcal{Z}_{s}}\left(\mathcal{X}_{s}, \mathcal{B}_{s} ; \mathcal{H}_{s}\right)=\operatorname{lct}_{Z}(X, B ; D)$. We conclude by Corollary 1.10 .

In particular, $\operatorname{lct}_{Z}(X, B ; \cdot)$ attains the maximal (resp. minimal) value on a dense open (resp. special closed) subset of $\Lambda$. Define

$$
\gamma_{Z}(X, B ; \Lambda)=\min _{D \in \Lambda} \operatorname{lct}_{Z}(X, B ; D) .
$$

If $X / k$ is proper, denote $\gamma_{X}(X, B ; \Lambda)$ by $\gamma(X, B ; \Lambda)$. Define similarly $\mu_{Z}(X, B ; \Lambda)$ and $\mu(X, B ; \Lambda)$

Theorem 2.7. Let $\left(X_{i} / k, B_{i}\right)$ be finitely many proper log varieties, with log canonical singularities. Let $\left|L_{i}\right|$ be non-empty complete linear systems on $X_{i}$. Let $X=\prod_{i} X_{i}, B=\sum_{i} p_{i}^{*}\left(B_{i}\right), L=$ $\sum_{i} p_{i}^{*}\left(L_{i}\right)$. Then the product log variety $(X, B)$ has log canonical singularities, the complete linear system $|L|$ is non-empty, and

$$
\gamma(X, B ;|L|)=\min _{i} \gamma\left(X_{i}, B_{i} ;\left|L_{i}\right|\right) .
$$

Proof. By induction, suffices to consider only two factors.

Let $D_{i} \in\left|L_{i}\right|$, for $i=1,2$. Let $t_{i} \geq 0$ be maximal such that $\left(X_{i}, B_{i}+t_{i} D_{i}\right)$ has $\log$ canonical singularities. Set $t=\min \left(t_{1}, t_{2}\right)$ and $D=p_{1}^{*} D_{1}+p_{2}^{*} D_{2}$. Then $D \in|L|$ and $t$ is maximal such that $(X, B+t D)$ has $\log$ canonical singularities. Therefore $\gamma \leq \operatorname{lct}(X, B ; D)=t$. Taking minimum after all members, we obtain $\gamma(X, B ;|L|) \leq \min _{i} \gamma\left(X_{i}, B_{i} ;\left|L_{i}\right|\right)$.

Suppose by contradiction that $\gamma(X, B ;|L|)<\min _{i} \gamma\left(X_{i}, B_{i} ;\left|L_{i}\right|\right)$. That is, there exists $D \in|L|$ and $t \leq \min _{i} \gamma\left(X_{i}, B_{i} ;\left|L_{i}\right|\right)$ such that $(X, B+t D)$ does not have log canonical singularities. Denote $Z=(X, B+t D)_{-\infty}$. It is a proper subset of $X$. If we show that $Z=\pi_{i}^{-1} \pi_{i}(Z)$ for every $i$, it follows that $Z=\emptyset$, a contradiction.

We prove the claim for the second projection $\pi: X \rightarrow X_{2}$. Choose a closed point $P \in X_{2}$ and suppose $X_{P} \not Z Z$. We will show that $X_{P} \cap Z=\emptyset$. Indeed, by Hironaka's flattening, there exists a desingularization $g: X_{2}^{\prime} \rightarrow X$ such that the induced Cartier divisor $D^{\prime}$ on $X^{\prime}=X_{1} \times X_{2}^{\prime}$ admits a decomposition $D^{\prime}=D^{\prime \prime}+\pi^{*}\left(D_{2}\right)$, where $D^{\prime \prime}$ is an effective Cartier divisor on $X^{\prime}$ relative over $X_{2}^{\prime}$, and $D_{2}$ is an effective Cartier divisor on $X_{2}^{\prime}$ with normal crossing support.

Choose a point $Q \in g^{-1}(P)$. Since $\left.D^{\prime \prime}\right|_{X_{Q}^{\prime}} \in\left|L_{1}\right|$ and $t \leq \gamma\left(X_{1}, B_{1} ;\left|L_{1}\right|\right)$, the log variety $\left(X_{1}, B_{1}+\right.$ $\left.\left.t D^{\prime \prime}\right|_{X_{1} \times Q}\right)$ has $\log$ canonical singularities. This is the fiber at $Q$ of the family of $\log$ varieties $\left(X^{\prime} / X_{2}^{\prime}, p_{1}^{*}\left(B_{1}\right)+t D^{\prime \prime}\right)$, so by Lemma 1.7, the log variety $\left(X^{\prime}, p_{1}^{*}\left(B_{1}\right)+t D^{\prime \prime}+\pi^{\prime *} \Sigma_{Q}\right)$ has $\log$ canonical singularities near $X_{Q}^{\prime}$, where $\Sigma_{Q}$ is any local divisor cut out by a regular system of parameters of $\mathcal{O}_{X_{2}^{\prime}, Q}$

On the other hand, $(X, B+t D)$ has log canonical singularities at the generic point of $X_{P}$. Therefore $\left(X^{\prime}, B^{\prime}+t D^{\prime}\right)$ has log canonical singularities at the generic point of $X_{Q}^{\prime}$. Let $g^{*}\left(K_{X_{2}}+\right.$ $\left.B_{2}\right)=K_{X_{2}^{\prime}}+B_{2}^{\prime}$. It follows that $\left(X_{2}^{\prime}, B_{2}^{\prime}+t D_{2}\right)$ has $\log$ canonical singularities. Therefore $B_{2}^{\prime}+t D_{2}^{\prime} \leq$ $\Sigma_{Q}$ for some choice of local parameters at $Q$. We deduce that $\left(X^{\prime}, p_{1}^{*}\left(B_{1}\right)+t D^{\prime \prime}+\pi^{\prime *}\left(B_{2}^{\prime}+t D_{2}^{\prime}\right)\right)$ has $\log$ canonical singularities near $X_{Q}^{\prime}$. That is $\left(X^{\prime}, B^{\prime}+t D^{\prime}\right)$ has log canonical singularities near $X_{Q}^{\prime}$. This holds for every $Q \in g^{-1}(P)$, so we deduce that $\left(X^{\prime}, B^{\prime}+t D^{\prime}\right)$ has log canonical singularities over an open neighborhood of $g^{-1}(P)$. Since $\left(X^{\prime}, B^{\prime}+t D^{\prime}\right) \rightarrow(X, B+t D)$ is log crepant, it follows that $(X, B+t D)$ has $\log$ canonical singularities near $X_{P}$, that is $Z \cap X_{P}=\emptyset$.

Graded case. Let $L$ be a $\mathbb{Q}$-Cartier divisor on $X$ such that $|n L| \neq \emptyset$ for some $n \geq 1$. Let $x \in(X, B)_{l c}$ be a scheme point. The $\alpha$-invariant at $x$ of $L$ with respect to $(X, B)$ is defined as

$$
\gamma_{x}(X, B ; L)=\inf \left\{\operatorname{lct}_{x}\left(X, B ; \frac{D_{n}}{n}\right) ; n \geq 1, D_{n} \in|n L|\right\}
$$


Question 2.8. Is $(X, B)_{l c} \ni x \mapsto \gamma_{x}(X, B ; L)$ is lower semicontinuous, with finitely many rational values?

Let $m \geq 1$ such that $m L$ is Cartier and $|m L| \neq \emptyset$. Then $\gamma_{x}(X, B ; L)=\inf _{m \mid n} n \gamma_{x}(X, B ;|n L|)$.

Lemma 2.9. If $\operatorname{dim} X=1, \operatorname{deg} L>0$ and $x \in X$ is a closed point, then $\gamma_{x}(X, B ; L)=\frac{1-b_{x}}{\operatorname{deg} L}$.

Proof. We may suppose $b_{x}<1$. Let $m L$ be Cartier and $|m L| \neq \emptyset$. Let $g$ be the genus of $C$. Recall that any complete linear system of degree $g$ is non-empty. Let $m \mid n$ and $n \operatorname{deg} L>g$. Then $|n L-(n \operatorname{deg} L-g) x| \neq \emptyset$. Therefore

$$
n \frac{\operatorname{deg} L}{1-b_{x}}-\frac{g}{1-b_{x}} \leq \mu_{x}(X, B ;|n L|) \leq n \frac{\operatorname{deg} L}{1-b_{x}} .
$$

Dividing by $n$ and letting $n \rightarrow \infty$, we obtain the claim.

Proposition 2.10 ([13], Theorem 6.7.1). Suppose $X^{d}$ is proper and $L$ is a nef and big $\mathbb{Q}$-divisor. Then $\gamma_{P}(X, B ; L) \cdot \sqrt[d]{\left(L^{d}\right)} \leq d$ for every $P \in(X, B)_{l c}$.

Proof. We may scale $L$ and suppose it is Cartier. Let $0<c<\sqrt[d]{\left(L^{d}\right)}$ be a rational number. Since $h^{0}(n L)=\left(L^{d}\right) \frac{n^{d}}{d !}+O\left(n^{d-1}\right)$ and $\left(\begin{array}{c}n c+d \\ d\end{array}\right)=c^{d} \frac{n^{d}}{d !}+O\left(n^{d-1}\right)$, there exists an integer $n \geq 1$ such that $n c \in \mathbb{Z}$ and $h^{0}(n L)>\left(\begin{array}{c}n c+d \\ d\end{array}\right)$.

Let $Q \in X \backslash(\operatorname{Sing} X \cup \operatorname{Supp} B)$. The evaluation map $\Gamma(X, n L) \rightarrow \mathcal{O}_{Q} / \mathfrak{m}_{Q}^{n c+1}$ has nontrivial kernel, by dimension count. Therefore there exists $D \in|n L|$ such that $\operatorname{mult}_{Q}(D)>n c$. Let $(X, B+\gamma D)$ be maximally $\log$ canonical at $Q$. Let $v$ be the valuation induced by the exceptional divisor of the blow-up of $Q \in X$. Then $0 \leq a(v ; X, B+\gamma D)=d-\gamma$ mult $_{v}(D)<d-\gamma n c$. Therefore $\gamma<\frac{d}{n c}$. We conclude $\gamma_{Q}(X, B ;|n L|)<\frac{d}{n c}$.

Since the points $Q$ are dense in $X$, Theorem 2.5 gives $\gamma_{P}(X, B ;|n L|)<\frac{d}{n c}$ for every $P \in(X, B)_{l c}$. Then $\gamma_{P}(X, B ; L) \leq n \gamma_{P}(X, B ;|n L|)<\frac{d}{c}$ for every $P \in(X, B)_{l c}$. Letting $c$ converge to $\sqrt[d]{\left(L^{d}\right)}$, we obtain the claim.

If $X / k$ is proper and $(X, B)$ has $\log$ canonical singularities, define the $\alpha$-invariant of $L$ with respect to $(X, B)$ as

$$
\gamma(X, B ; L)=\inf \left\{\operatorname{lct}\left(X, B ; \frac{D_{n}}{n}\right) ; n \geq 1, D_{n} \in|n L|\right\} .
$$

Question 2.11. Does $\operatorname{mld}(X, B)>0$ imply $\gamma(X, B ; L)>0$ ?

For example, $\gamma(X, B ; L)=\frac{\operatorname{mld}(X, B)}{\operatorname{deg} L}$ if $X$ is a curve and $L \chi_{\mathbb{Q}} 0$ (by Lemma 2.9). If $X$ is smooth, $B=0$, and $A$ is a very ample divisor on $X$, the argument of [17, Corollary 5.11] shows that $\gamma(X, 0 ; L) \geq \frac{1}{\left(L \cdot A^{d-1}\right)}$. Theorem 2.7 gives

Corollary 2.12. Let $\left(X_{i} / k, B_{i}\right)$ be finitely many proper log varieties, with log canonical singularities. Let $L_{i}$ be $\mathbb{Q}$-Cartier divisors such that $\left|m L_{i}\right| \neq \emptyset$ for some $m \geq 1$. Let $X=\prod_{i} X_{i}, B=\sum_{i} p_{i}^{*}\left(B_{i}\right)$, $L=\sum_{i} p_{i}^{*}\left(L_{i}\right)$. Then the product $\log$ variety $(X, B)$ has $\log$ canonical singularities and

$$
\gamma(X, B ; L)=\min _{i} \gamma\left(X_{i}, B_{i} ; L_{i}\right)
$$

The $\alpha$-invariant in terms of width. Let $X$ be a normal variety. Let $\Lambda$ be a non-empty, finite dimensional linear system on $X$. For a prime divisor $E \subset X$, define the width of $\Lambda$ at $E$ as

$$
w_{E}(\Lambda)=\sup \left\{\operatorname{mult}_{E}(D) ; D \in \Lambda\right\} .
$$

It is a non-negative integer, zero if and only if $E$ is not a fixed component of $\Lambda$ and $\phi_{\Lambda}(E)=\phi_{\Lambda}(X)$. If $f: X^{\prime} \rightarrow X$ is a proper modification and $E \subset X^{\prime}$ is a prime divisor, define the width of $\Lambda$ at $E$ as $w_{E}\left(f^{*} \Lambda\right)$. It depends only on the valuation of $X$ defined by $E$ (called geometric valuation of $X$ ). 
Let $L$ be a $\mathbb{Q}$-Cartier divisor such that $m L$ is Cartier and $|m L| \neq \emptyset$ for some $m \geq 1$. Let $E$ be a geometric valuation of $X$. Define the width of $L$ at $E$ as

$$
w_{E}(L)=\sup \left\{\frac{w_{E}(|n L|)}{n} ; m \mid n\right\}
$$

If $X$ is proper, $w_{E}(L)=0$ for every geometric valuation $E$ of $X$ if and only if $L \sim_{\mathbb{Q}} 0$. If $X$ is projective of dimension $d, A$ is a very ample divisor on $X$, and $E$ is a prime divisor on $X$, then $w_{E}(L) \leq\left(L \cdot A^{d-1}\right)$. It follows that if $X$ is proper, then $w_{E}(L)$ is a non-negative real number, for every geometric valuation $E$ of $X$. By definition, the following formulas hold:

- Let $(X, B)$ be a log variety, $\Lambda$ a non-empty finite dimensional linear system on $X$. Let $E$ be a geometric valuation of $(X, B)_{l c}$. Then

$$
\gamma_{E}(X, B ; \Lambda)= \begin{cases}+\infty & , w_{E}(\Lambda)=0 \\ \frac{a(E ; X, B)}{w_{E}(\Lambda)} & , w_{E}(\Lambda)>0\end{cases}
$$

- Let $(X, B)$ be a proper log variety, with $\log$ canonical singularities. Let $L$ be a $\mathbb{Q}$-Cartier divisor such that $m L$ is Cartier and $|m L| \neq \emptyset$ for some $m \geq 1$. Then $\gamma(X, B ; L)$ is the infimum of $\gamma_{E}(X, B ; L)$ after all geometric valuations $E$ of $X$. Equivalently,

$$
\gamma(X, B ; L)= \begin{cases}+\infty & , L \sim_{\mathbb{Q}} 0 \\ \inf _{w_{E}(L)>0} \frac{a(E ; X, B)}{w_{E}(L)} & , L \chi_{\mathbb{Q}} 0\end{cases}
$$

\section{THE $\alpha$-INVARIANT ON TORIC VARIETIES}

Let $X / k$ be a proper toric variety, let $B$ be an effective $\mathbb{Q}$-divisor which is torus invariant, such that $K_{X}+B$ is $\mathbb{Q}$-Cartier and $(X, B)$ has at most log canonical singularities. Due to the existence of $\log$ resolutions in the toric category, the latter condition is equivalent to $B=\sum_{i} b_{i} E_{i}$, where $E_{i}$ are the torus invariant prime divisors of $X$ and $b_{i} \in[0,1] \cap \mathbb{Q}$. We have $a\left(E_{i} ; X, B\right)=1-b_{i}$.

Let $L=\sum_{i} l_{i} E_{i}$ be a torus invariant $\mathbb{Q}$-Cartier divisor. Recall that $\Gamma(X, L)=\bigoplus_{m \in M \cap \square_{L}} k \cdot \chi^{m}$, where $\square_{L}=\cap_{i}\left\{m \in M_{\mathbb{R}} ;\left\langle m, e_{i}\right\rangle+l_{i} \geq 0\right\}$ and $\left\{e_{i}\right\}=\Delta_{X}(1)$.

Let $V \subseteq \Gamma(X, L)$ be a non-zero, torus invariant $k$-vector subspace. There exists a finite set $A \subseteq M \cap \square_{L}$ such that $V=\oplus_{m \in A} k \cdot \chi^{m}$. Let $\Lambda=\{(f)+L ; f \in V \backslash 0\}$ be the corresponding linear system.

Lemma 3.1. $w_{E_{i}}(\Lambda)$ is attained by a torus invariant member, computed by the formula:

$$
w_{E_{i}}(\Lambda)=\max _{m \in A}\left\langle m, e_{i}\right\rangle+l_{i} .
$$

Proof. Let $t \geq 0$. The set $\left\{f \in V ; \operatorname{mult}_{E_{i}}((f)+L) \geq t\right\}$ is a torus invariant vector subspace of $V$. So it is non-zero if and only if it contains $\chi^{m}$ for some $m \in A$. It follows that the maximal (also minimal) value among $\operatorname{mult}_{E_{i}}(D)(D \in \Lambda)$ is attained within the subset $\operatorname{mult}_{E_{i}}\left(\left(\chi^{m}\right)+L\right)=$ $\left\langle m, e_{i}\right\rangle+l_{i}(m \in A)$.

We have $w_{E_{i}}(\Lambda)=0$ if and only if $A$ is contained in the hyperplane $\left\langle\cdot, e_{i}\right\rangle+l_{i}=0$. It follows that we can compute $\gamma_{x}(X, B ; \Lambda)$ for every torus invariant codimension one point $x \in X$. Indeed, $x$ is the generic point of some $E_{i}$, and $\gamma_{E_{i}}(X, B ; \Lambda)$ is $+\infty$ if $w_{E_{i}}(\Lambda)=0$, and $\frac{1-b_{i}}{\max _{m \in A}\left\langle m, e_{i}\right\rangle+l_{i}}$ otherwise. Proposition 3.2 states that only these valuations determine $\gamma(X, B ; \Lambda)$.

Proposition 3.2. $\gamma(X, B ; \Lambda)=\min _{i} \gamma_{E_{i}}(X, B ; \Lambda)$. In particular, $\gamma(X, B ; \Lambda)$ is attained by a torus invariant member of $\Lambda$.

Proof. The inequality $\leq$ is clear. For the converse, let $t \leq \min _{i} \gamma_{E_{i}}(X, B ; \Lambda)$ and $D \in \Lambda$. We have to show that $(X, B+t D)$ has log canonical singularities. 
Let $\mu: X^{\prime} \rightarrow X$ be a toric birational modification which is an isomorphism in codimension one, and such that $X^{\prime}$ is $\mathbb{Q}$-factorial. The toric varieties $X, X^{\prime}$ have the same invariant prime divisors, and therefore we may pullback our data to $X^{\prime}$. Therefore we may suppose $X$ is $\mathbb{Q}$-factorial.

The conclusion is local on $X$, so we may shrink $X$ to a torus invariant affine open neighborhood $U$ of a fixed point. Thus $U=T_{N} \operatorname{emb}(\sigma)$, where $\sigma$ is a simplicial cone in $N_{\mathbb{R}}$ which generates $N_{\mathbb{R}}$. Let $N^{\prime}$ be the lattice generated by the primitive vectors $e_{i} \in N$ which generate the extremal rays of $\sigma$. The inclusion $N^{\prime} \subseteq N$ induces a toric morphism $\tau: U^{\prime} \rightarrow U$ which is finite, and étale in codimension one. We have $\left(U^{\prime}, U^{\prime} \backslash T\right) \simeq\left(\mathbb{A}_{k}^{d}, \sum_{j=1}^{d} H_{j}\right)$, where $H_{i}$ are the standard hyperplanes of the affine space. We have $\tau^{*}\left(K_{X}+B+\left.t D\right|_{U}\right)=K_{\mathbb{A}_{k}^{d}}+\sum_{j=1}^{d} b_{j} H_{j}+t D^{\prime}$. We may suppose $\left\lfloor\tau^{*} L\right\rfloor=0$, and therefore $\left(\mathbb{A}_{k}^{d}, \sum_{j=1}^{d} b_{j} H_{j}+t D^{\prime}=\sum_{j=1}^{d} b_{j} H_{j}+t\left\{D^{\prime}\right\}+t\left\lfloor D^{\prime}\right\rfloor\right)$ has log canonical singularities by Lemma 3.3. Therefore $(X, B+t D)$ has $\log$ canonical singularities on $U$.

Lemma 3.3. Let $H_{i}$ be the standard hyperplanes of the affine space $\mathbb{A}_{k}^{d}$. Let $b_{i} \in[0,1], t>0$, and $0 \neq P \in k\left[z_{1}, \ldots, z_{d}\right]$ such that $\operatorname{deg}_{z_{i}}(P) \leq \frac{1-b_{i}}{t}$ for every $1 \leq i \leq d$. Let $D$ be the divisor of zeros of $P$. Then $\left(\mathbb{A}_{k}^{d}, \sum_{i=1}^{d} b_{i} H_{i}+t D\right)$ has log canonical singularities.

Proof. Denote $w_{i}=\operatorname{deg}_{z_{i}}(P)$. Consider the product of $\log$ varieties $\prod_{i=1}^{d}\left(\mathbb{P}^{1}, b_{i} \cdot 0\right)$. We have $\gamma\left(\mathbb{P}^{1}, b_{i} \cdot 0 ;\left|\mathcal{O}\left(w_{i}\right)\right|\right)=\frac{1-b_{i}}{w_{i}} \geq t$. By Theorem 2.7, $\gamma\left(\prod_{i=1}^{d}\left(\mathbb{P}^{1}, b_{i} \cdot 0\right) ;\left|\mathcal{O}\left(w_{1}, \ldots, w_{d}\right)\right|\right) \geq t$.

Now $P$ defines a divisor $D^{\prime} \in\left|\mathcal{O}\left(w_{1}, \ldots, w_{d}\right)\right|$. Therefore $\left(\prod_{i} \mathbb{P}^{1}, \bigotimes_{i} b_{i} \cdot 0+t D^{\prime}\right)$ has log canonical singularities. After restricting to the complement of $\bigotimes_{i} \infty$, we obtain that $\left(\mathbb{A}_{k}^{d}, \sum_{i=1}^{d} b_{i} H_{i}+t D\right)$ has log canonical singularities.

Suppose now that $|n L| \neq \emptyset$ for some $n \geq 1$, that is $\square_{L} \neq \emptyset$. The width of $L$ in $E_{i}$ is computed by the formula

$$
w_{E_{i}}(L)=\max _{m \in \square_{L}}\left\langle m, e_{i}\right\rangle+l_{i}
$$

Let $r \geq 1$ be the smallest integer such that the extremal points of $r \square_{L}$ belong to the lattice $M$ (i.e. $\Gamma(r L) \otimes \Gamma(n L) \rightarrow \Gamma((r+n) L)$ is surjective for $n \gg 0)$. Then $w_{E_{i}}(L)=\frac{w_{E_{i}}(|r L|)}{r}$.

The width of $L$ in $E_{i}$ is zero if and only if $\square_{L}$ is contained in the hyperplane $\left\langle\cdot, e_{i}\right\rangle+l_{i}=0$. In this case, $\gamma_{E_{i}}(X, B ; L)=+\infty$. If $w_{E_{i}}(L)>0$, then

$$
\gamma_{E_{i}}(X, B ; L)=\frac{1-b_{i}}{w_{E_{i}}(L)} .
$$

Proposition 3.2 for the complete linear systems $|n L|$ gives

Theorem 3.4. $\gamma(X, B ; L)=\min _{i} \gamma_{E_{i}}(X, B ; L)$. In particular, $\gamma(X, B ; L)$ is attained by some invariant member $\left(\chi^{m}\right)+L\left(m \in M_{\mathbb{Q}} \cap \square_{L}\right)$.

Recall the the stable fixed multiplicity of $L$ in $E_{i}$ is defined as

$$
f_{E_{i}}(L)=\inf \left\{\frac{\operatorname{mult}_{E_{i}}\left(D_{n}\right)}{n} ; n \geq 1, D_{n} \in|n L|\right\} .
$$

In our toric setting, it has the following combinatorial formula:

$$
f_{E_{i}}(L)=\min _{m \in \square_{L}}\left\langle m, e_{i}\right\rangle+l_{i}
$$

It is zero if and only if $E_{i}$ is not fixed by $|n L|$ for some $n \geq 1$. More precisely, $r f_{E_{i}}(L)=$ mult $_{E_{i}} \operatorname{Fix}(|r L|)$, where $r$ is defined above.

Recall that the width of a convex set $\square \subset M_{\mathbb{R}}$ along a direction $e \in N_{\mathbb{R}} \backslash 0$ is defined as $w(\square ; e)=\sup \left\{\left\langle m^{\prime}, e\right\rangle-\langle m, e\rangle ; m^{\prime}, m \in \square\right\}$. We obtain the identity:

$$
w\left(\square_{L} ; e_{i}\right)=w_{E_{i}}(L)-f_{E_{i}}(L) .
$$


Corollary 3.5. Let $(X, B)$ be a proper log variety with log canonical singularities. Let $L$ be a torus invariant $\mathbb{Q}$-Cartier divisor such that the linear system $|n L|$ is mobile for some $n \geq 1$. Then

$$
\gamma(X, B ; L)=\sup \left\{t \geq 0 ; t\left(\square_{L}-\square_{L}\right) \subseteq \square_{-K_{X}-B}\right\} .
$$

Proof. We have $t\left(\square_{L}-\square_{L}\right) \subseteq \square_{-K_{X}-B}$ if and only if $t\left(\square_{L}-m\right) \subseteq \square_{-K_{X}-B}$ for every $m \in \square_{L}$. Since $-K_{X}-B=\sum_{i}\left(1-b_{i}\right) E_{i}$, this is equivalent to $t\left\langle m^{\prime}-m, e_{i}\right\rangle+1-b_{i} \geq 0$ for every invariant prime divisor $E_{i} \subset X$, and $m, m^{\prime} \in \square_{L}$. Equivalently, $t \cdot w\left(\square_{L} ; e_{i}\right) \leq 1-b_{i}$ for every $i$. By assumption, $f_{E_{i}}(L)=0$ for every $i$. That is $w\left(\square_{L} ; e_{i}\right)=w_{E_{i}}(L)$. The condition becomes $t \cdot w_{E_{i}}(L) \leq 1-b_{i}$ for every $i$, that is $t \leq \gamma(X, B ; L)$, by Theorem 3.4 .

Lemma 3.6. Let $(X, B)$ be a proper toric log variety, with log canonical singularities. Let $L$ be a torus invariant $\mathbb{Q}$-Cartier divisor such that $|n L| \neq \emptyset$ for some $n \geq 1$.

a) $X \ni P \mapsto \gamma_{P}(X, B ; L)$ is lower semicontinuous, and takes only finitely many values.

b) Let $P \in X \backslash T$ be a closed point outside the torus. Let $O$ be the generic point of the unique torus orbit which contains $P$. Then $\gamma_{P}(X, B ; L)=\gamma_{O}(X, B ; L)$.

Proof. a) Fix $t>0$. Then $\left\{P \in X ; \gamma_{P}(X, B ; L)<t\right\}=\cup_{n \geq 1} Z(t, n)$, where $Z(t, n)=\cup_{D_{n} \in|n L|}(X, B+$ $\left.\frac{t}{n} D_{n}\right)_{-\infty}$. Each $Z(t, n)$ is torus invariant. And is closed by Theorem 1.8 applied to the universal divisor of $|n L|$. Since $X$ contains only finitely many closed torus invariant subsets, $Z(t, n)$ belong to a finite set. Therefore $\cup_{n} Z(t, n)$ is closed in $X$. We conclude that $P \mapsto \gamma_{P}(X, B ; L)$ is lower semicontinuous. The function is constant on the torus orbits, and since $X$ has only finitely many orbits, we conclude that the function takes only finitely many values.

b) The inequality $\leq$ is clear. For the converse, let $t \leq \gamma_{O}(X, B ; L)$. Then $Z(t, n)$ is a closed torus invariant subset of $X$ which does not contain the generic point of the orbit $O$. Therefore $Z(t, n)$ is disjoint from $O$. Therefore $t \leq \gamma_{P}(X, B ; L)$.

\section{Hensley type Diophantine approximation}

Geometry of numbers [14]. Let $V \simeq \mathbb{R}^{d}$ be a finite dimensional $\mathbb{R}$-vector space. Let $V^{*}$ be the dual vector space. The dual of a non-empty convex set $\square \subseteq V$ is defined as

$$
\square^{*}=\left\{v^{*} \in V^{*} ;\left\langle v^{*}, v\right\rangle+1 \geq 0 \forall v \in \square\right\} .
$$

It is closed, convex subset of $V^{*}$, containing the origin. The Duality Theorem states that if $\square$ is compact convex and contains the origin in its interior, then $\left(\square^{*}\right)^{*}=\square$.

Let $\square \subset V$ be a compact convex set. Let $P \in \square$ be a point. Denote

$$
\gamma(P \in \square)=\sup \{t \geq 0 ; P+t(\square-\square) \subseteq \square\}
$$

It is a well defined non-negative real number, zero if and only if $P$ does not belong to the relative interior of $\square$. We can think of $\gamma(\cdot \in \square)$ as a distance function to the boundary of $\square$.

Suppose $\operatorname{dim} \square=\operatorname{dim} V$ and $P$ is an interior point of $\square$. For each $v \in V \backslash 0$, there exist $l^{+}, l^{-}>0$ such that $P+l^{+} v, P-l^{-} v \in \partial \square$. The supremum of the ratio $l^{+} / l^{-}$, after directions $v \in V \backslash 0$, is called the coefficient of asymmetry of $\square$ about $P$, denoted $c(P \in \square)$. We have

$$
\gamma(P \in \square)=\frac{1}{1+c(P \in \square)}
$$

By definition, $c(P \in \square) \geq 1$. Therefore $\gamma(P \in \square) \leq \frac{1}{2}$, and equality holds if and only if $\square$ is symmetric about $P$.

If 0 is an interior point of a compact convex set $\square$, then so is $0 \in \square^{*}$, and $\gamma(0 \in \square)=\gamma\left(0 \in \square^{*}\right)$.

Suppose $\square$ is a compact polytope and $P$ is an interior point. To compute $c(P \in \square)$ it suffices to consider the directions $v$ such that $P+\mathbb{R} v$ contains some vertex of $\square$. In particular, the supremum in the definition of $c(P \in \square)$ is a maximum. And if both $P$ and $\square$ are rational with respect to 
some lattice $\Lambda$ with $\Lambda \otimes_{\mathbb{Z}} \mathbb{R}=V$, then $c(P \in \square) \in \mathbb{Q}$. Fixing a line which attains the maximum and passes through a vertex of $\square$, we can apply Caratheodory's Theorem to the other boundary point, and obtain the following statement (called the simplex trick): there exists a simplex $S$ (with $\operatorname{dim} S \leq \operatorname{dim} V)$, with vertices among those of $\square$, containing $P$ in its relative interior, and with $c(P \in \square) \leq c(P \in S)$.

If $S$ is a simplex and $P$ has barycentric coordinates $\left(\gamma_{i}\right)_{i}$ with respect to the vertices of $S$, then $\gamma(P \in S)=\min _{i} \gamma_{i}$.

Van der Corput's theorem gives

Theorem 4.1. Let $\square \subset \mathbb{R}^{d}$ be a compact convex set, containing the origin in the interior. Then

$$
\left|\mathbb{Z}^{d} \cap \operatorname{int}(\square)\right| \geq \gamma(0 \in \square)^{d} \operatorname{vol}_{\mathbb{Z}^{d}}(\square) .
$$

The original Minkowski's first theorem asserts that if $\square$ is symmetric about the origin, that is $\gamma(0 \in \square)=\frac{1}{2}$, then $\{0\} \subsetneq \mathbb{Z}^{d} \cap \operatorname{int}(\square)$ if $\operatorname{vol}_{\mathbb{Z}^{d}}(\square)>2^{d}$.

Diophantine approximation. For positive integers $p, q$, define integers $u_{p, q}$ recursively as follows: $u_{1, q}=q, u_{p+1, q}=u_{p, q}\left(1+u_{p, q}\right)$. The following properties hold:

- $q \mid u_{p, q}$ and $\operatorname{gcd}\left(1+u_{p, q}, 1+u_{p^{\prime}, q}\right)=1$ for $p \neq p^{\prime}$.

- $\sum_{i=1}^{p} \frac{1}{1+u_{i, q}}=\frac{1}{q}-\frac{1}{u_{p+1, q}}$. In particular, $\sum_{i=1}^{\infty} \frac{1}{1+u_{i, q}}=\frac{1}{q}$.

- $\prod_{i=1}^{p}\left(1+u_{i, q}\right)=\frac{u_{p+1, q}}{q}$.

- $\prod_{i=1}^{p} \frac{1}{1+u_{i, q}}=1-q \sum_{i=1}^{p} \frac{1}{1+u_{i, q}}$.

Note that $\left(1+u_{p, 1}\right)_{p \geq 1}=(2,3,7,43, \ldots)$ is called the Sylvester sequence in the literature. And $u_{p, q}$ can be expressed as a polynomial in $q$, with leading term $q^{2^{p-1}}$.

Lemma 4.2. 15] Let $d$ be a positive integer. Let $x_{1} \geq \cdots \geq x_{d}>0$ and $y_{1} \geq \cdots \geq y_{d}>0$ be real numbers such that $\prod_{i=1}^{l} x_{i} \geq \prod_{i=1}^{l} y_{i}$ for every $1 \leq l \leq d$. Then $\sum_{i=1}^{d} x_{i} \geq \sum_{i=1}^{d} y_{i}$, and equality holds if and only if $x_{i}=y_{i}$ for every $i$.

Lemma 4.3 (cf. [15]). Let $d, q$ be positive integers. Let $x_{1} \geq \ldots \geq x_{d}>0$ be real numbers such that $\prod_{i=1}^{l} x_{i} \leq 1-q \sum_{i=1}^{l} x_{i}$ for every $1 \leq l \leq d$. Then

$$
\sum_{i=1}^{d} x_{i} \leq \sum_{i=1}^{d} \frac{1}{1+u_{i, q}}
$$

and equality holds if and only if $x_{i}=\frac{1}{1+u_{i, q}}$ for every $i$.

Proof. Denote $y_{i}=\frac{1}{1+u_{i, q}}$. We have $x_{1} \leq 1-q x_{1}$, that is $x_{1} \leq y_{1}$. Therefore there exists $1 \leq k \leq d$ maximal such that $\prod_{i=1}^{l} x_{i} \leq \prod_{i=1}^{l} y_{i}$ for every $1 \leq l \leq k$.

Suppose $k=d$. Lemma 4.2 gives $\sum_{i=1}^{d} x_{i} \leq \sum_{i=1}^{d} y_{i}$. And equality holds if and only if $x_{i}=y_{i}$ for every $i$.

Suppose $k<d$. Then $\prod_{i=1}^{k+1} x_{i}>\prod_{i=1}^{k+1} y_{i}$. It follows that $\prod_{i=j}^{k+1} x_{i}>\prod_{i=j}^{k+1} y_{i}$ for $1 \leq j \leq k+1$. Lemma 4.2 gives $\sum_{i=1}^{k+1} x_{i}>\sum_{i=1}^{k+1} y_{i}$. Therefore

$$
\prod_{i=1}^{k+1} x_{i} \leq 1-q \sum_{i=1}^{k+1} x_{i}<1-q \sum_{i=1}^{k+1} y_{i}=\prod_{i=1}^{k+1} y_{i} .
$$

Contradiction. 
Lemma 4.4. For indeterminates $T_{1}, \ldots, T_{d}$, the following formula holds:

$$
\operatorname{det}\left(\begin{array}{cccc}
1+T_{1} & 1 & & 1 \\
1 & 1+T_{2} & & 1 \\
\vdots & & \ddots & \vdots \\
1 & \ldots & 1 & 1+T_{d}
\end{array}\right)=\left(1+\sum_{i=1}^{d} \frac{1}{T_{i}}\right) \prod_{i=1}^{d} T_{i}
$$

Proof. We use induction on $d$. The case $d=1$ is clear. Let $d \geq 2$. The determinant is of the form $c_{1} T_{1}+c_{0}$, where $c_{0}, c_{1}$ are polynomials in $T_{2}, \ldots, T_{d}$. The constant term is obtained by setting $T_{1}=0$, and we compute $c_{0}=\prod_{i=2}^{d} T_{i}$. The other term is the difference

$$
\operatorname{det}\left(\begin{array}{cccc}
1+T_{1} & 1 & & 1 \\
1 & 1+T_{2} & & 1 \\
\vdots & & \ddots & \vdots \\
1 & \ldots & 1 & 1+T_{d}
\end{array}\right)-\operatorname{det}\left(\begin{array}{cccc}
T_{1} & 1 & & 1 \\
1 & 1+T_{2} & & 1 \\
\vdots & & \ddots & \vdots \\
1 & \ldots & 1 & 1+T_{d}
\end{array}\right)
$$

We compute the determinants using the formula by permutations $\sigma$ of $\{1, \ldots, d\}$. If $\sigma(1) \neq 1$, the corresponding difference is zero. Therefore $c_{1}=\left(1+T_{1}\right) \operatorname{det}_{d-1}-T_{1} \operatorname{det}_{d-1}=\operatorname{det}_{d-1}$. By induction, $c_{1}=\sum_{i=2}^{d} \prod_{j \neq i, 1} T_{j}+\prod_{i=2}^{d} T_{i}$. Then the determinant is $\sum_{i=2}^{d} \prod_{j \neq i} T_{j}+\prod_{i=1}^{d} T_{i}+\prod_{j \neq 1} T_{j}$, so the desired identity holds for $d$.

Lemma 4.5. Let $x_{1}, \ldots, x_{d}>0$ and $c_{1}, \ldots, c_{d} \geq 1$ such that $1-\prod_{i=1}^{d} x_{i}<\sum_{i=1}^{d} c_{i} x_{i}<1$. Then there exists $z \in \mathbb{N}^{d} \backslash 0$ such that $\frac{z_{j}}{1+\sum_{i} c_{i} z_{i}}<x_{j}$ for all $j$.

Proof. Consider the convex set $U=\left\{z \in \mathbb{R}^{d} ;\|A z\|_{\infty}<1\right\}$, where A is the $d \times d$ matrix

$$
\left(\begin{array}{cccc}
c_{1}-\frac{1}{x_{1}} & c_{2} & & c_{d} \\
c_{1} & c_{2}-\frac{1}{x_{2}} & & c_{d} \\
\vdots & & \ddots & \vdots \\
c_{1} & \cdots & c_{d-1} & c_{d}-\frac{1}{x_{d}}
\end{array}\right)
$$

and the norm $\|\cdot\|_{\infty}$ is the maximum absolute value of the components. By Lemma 4.4, we compute

$$
\operatorname{det} A=(-1)^{d} \frac{1-\sum_{i} c_{i} x_{i}}{\prod_{i} x_{i}}
$$

By assumption, $0<|\operatorname{det} A|<1$. Then $\operatorname{vol}_{\mathbb{Z}^{d}}(U)=\frac{2^{d}}{|\operatorname{det} A|}>2^{d}$. The convex body $U$ is symmetric about the origin. By Minkowski's first theorem, there exists $0 \neq z \in \mathbb{Z}^{d} \cap U$. That is $z \in \mathbb{Z}^{d} \backslash 0$ and $\left|\sum_{i} c_{i} z_{i}-\frac{z_{j}}{x_{j}}\right|<1$ for every $j$. We may suppose $\sum_{i} c_{i} z_{i} \geq 0$, after possibly replacing $z$ by $-z$.

The inequality $\sum_{i} c_{i} z_{i}-\frac{z_{j}}{x_{j}}<1$ gives $\frac{z_{j}}{x_{j}}>-1$, that is $z_{j}>-x_{j}$. Since $c_{i} \geq 1$, we obtain $\sum_{i=1}^{d} x_{i}<1$. In particular, $x_{j}<1$. Therefore $z_{j}>-1$, that is $z_{j} \geq 0$.

The other inequality $-1<\sum_{i} c_{i} z_{i}-\frac{z_{j}}{x_{j}}$ is equivalent to $\frac{z_{j}}{1+\sum_{i} c_{i} z_{i}}<x_{j}$.

The following statement is the effective version of [11, Lemma 2.4]. The case $q=1$ was obtained in [5, Theorem 1.1].

Theorem 4.6. Let $q$ be a positive integer, let $1 \leq c_{1}, \ldots, c_{d} \leq q$. Let $x_{1} \geq \ldots \geq x_{d}>0$ such that $\sum_{i=1}^{d} x_{i} \geq \sum_{i=1}^{d} \frac{q}{1+u_{i, q}}$. Suppose $x_{i} \neq \frac{q}{1+u_{i, q}}$ for some $i$. Then there exists $z \in \mathbb{N}^{d} \backslash 0$ such that $\frac{c_{j} z_{j}}{1+\sum_{i} c_{i} z_{i}}<x_{j}$ for every $j$.

Proof. We may suppose $\sum_{i=1}^{d} x_{i}<1$. By Lemma 4.3 for $\left(\frac{x_{i}}{q}\right)_{i}$, there exists $1 \leq l \leq d$ such that $\prod_{i=1}^{l} x_{i}>q^{l}\left(1-\sum_{i=1}^{l} x_{i}\right)$. In particular, $\prod_{i=1}^{l} x_{i}>\left(\prod_{i=1}^{l} c_{i}\right)\left(1-\sum_{i=1}^{l} x_{i}\right)$. 
By Lemma 4.5 for $\left(\frac{x_{i}}{c_{i}}\right)_{i}$, there exists $z \in \mathbb{N}^{l} \backslash 0$ such that $\frac{c_{j} z_{j}}{1+\sum_{i=1}^{l} c_{i} z_{i}}<x_{j}$ for all $1 \leq j \leq l$. Set $z_{j}=0$ for $j>l$. Then $z \in \mathbb{N}^{d} \backslash 0$ satisfies the claim.

Upper bound for coefficient of asymmetry. The case $q=1$ of the following result was obtained in [6, Theorem 2.1].

Theorem 4.7. Let $q$ be a positive integer. Let $N$ be a d-dimensional lattice and $S$ a simplex with vertices in $N$ such that $N \cap \operatorname{int}\left(\frac{1}{q} S\right)=\{0\}$. Then $\gamma(0 \in S) \geq \frac{q}{u_{d+1, q}}$, and equality holds if and only if there exists an isomorphism $N \simeq \mathbb{Z}^{d}$ which maps $S$ to the convex hull of $e_{0}, \ldots, e_{d}$, where $e_{1}, \ldots, e_{d}$ is the standard basis of $\mathbb{Z}^{d}$ and $e_{0}=-\sum_{i=1}^{d} \frac{u_{d+1, q}}{1+u_{i, q}} e_{i}$.

Proof. Let $v_{0}, \ldots, v_{d}$ be the vertices of $S$. Let $0=\sum_{i=0}^{d} \gamma_{i} v_{i}$ be the barycentric coordinates of the origin. Suppose $\gamma_{0}=\min _{i} \gamma_{i}$.

Suppose by contradiction that $\gamma(0 \in S)<\frac{q}{u_{d+1, q}}$. That is $\gamma_{0}<\frac{q}{u_{d+1, q}}$. Then $\sum_{i=1}^{d} \gamma_{i}>\sum_{i=1}^{d} \frac{q}{1+u_{i, q}}$. By Theorem 4.6, there exists $z \in \mathbb{N}^{d} \backslash 0$ such that $\frac{q z_{i}}{1+\sum_{i} q z_{i}}<\gamma_{i}(1 \leq i \leq d)$. Set $z_{0}=0$ and denote $|z|=\sum_{i} z_{i}$. We have

$$
-q \sum_{i} z_{i} v_{j}=\sum_{i}\left((1+q|z|) \gamma_{i}-q z_{i}\right) v_{i}
$$

On the right hand side, the coefficients of $v_{i}$ are positive, and add up to 1 . Therefore $-q \sum_{i} z_{i} v_{j} \in$ $\operatorname{int}(S)$. That is $-\sum_{i} z_{i} v_{i} \in N \cap \operatorname{int}\left(\frac{1}{q} S\right) \backslash 0$, a contradiction.

We conclude that $\gamma_{0} \geq \frac{q}{u_{d+1, q}}$. Suppose now that $\gamma_{0}=\frac{q}{u_{d+1, q}}$. The above arguments and Theorem 4.6 give $\gamma_{i}=\frac{q}{1+u_{i, q}}$ for $1 \leq i \leq d$. We obtain the barycentric coordinates

$$
0=\frac{q}{u_{d+1, q}} v_{0}+\sum_{i=1}^{d} \frac{q}{1+u_{i, q}} v_{i}
$$

In particular, $\sum_{i=1}^{d} \frac{q}{1+u_{i, q}}\left(v_{i}-v_{0}\right)=-v_{0} \in N$. Since $\left(1+u_{i, q}\right)_{i}$ are pairwise relatively prime, we obtain $\frac{q}{1+u_{i, q}}\left(v_{i}-v_{0}\right) \in N$ for every $i$. Since $q \mid u_{i, q}$, we deduce $v_{i}-v_{0}=\left(1+u_{i, q}\right) w_{i}$ for some $w_{i} \in N$. The vectors $w_{1}, \ldots, w_{d} \in N$ are linearly independent, and $v_{0}=-q \sum_{i=1}^{d} w_{i}$.

The inequality $\sum_{i=1}^{d} \frac{1}{1+u_{i, q}}<\frac{1}{q}$ implies $v_{0}+\sum_{i=1}^{d}(0,1] w_{i} \subset \operatorname{int}\left(\frac{1}{q} S\right)$. Let $x_{i} \in(0,1]$ such that $\sum_{i=1}^{d} x_{i} w_{i} \in N$. Then $v_{0}+\sum_{i=1}^{d} x_{i} w_{i} \in N \cap \operatorname{int}\left(\frac{1}{q} S\right)$. Therefore $v_{0}+\sum_{i=1}^{d} x_{i} w_{i}=0$. Therefore $x_{i}=1$ for all $i$. We conclude that $w_{1}, \ldots, w_{d}$ is a basis for the lattice $N$.

But $v_{i}=\left(1+u_{i, q}\right) w_{i}-\sum_{j=1}^{d} q w_{j}=\sum_{j} a_{i j} w_{j}(1 \leq i \leq d)$. By Lemma4.4, we compute $\operatorname{det}\left(a_{i j}\right)=1$. Therefore $v_{1}, \ldots, v_{d}$ is a basis of $N$. This induces an isomorphism $N \simeq \mathbb{Z}^{d}$ such that $v_{i}(1 \leq i \leq d)$ correspond to the standard basis $e_{i}(1 \leq i \leq d)$, and $v_{0}$ corresponds to $-\sum_{i=1}^{d} \frac{u_{d+1, q}}{1+u_{i, q}} e_{i}$.

The following is the sharp version of [11, Corollary 3.2]:

Theorem 4.8. Let $\square \subset \mathbb{R}^{d}$ be a compact polytope with vertices in $\mathbb{Z}^{d}$, of dimension d. Suppose $\mathbb{Z}^{d} \cap$ int $\square$ has cardinality $q \geq 1$. Then for every $P \in \mathbb{Z}^{d} \cap$ int $\square$ we have

$$
\gamma(P \in \square) \geq \frac{q}{u_{d+1, q}}
$$

Proof. Fix $P \in \square$. We may replace $\square$ by $\square-P$, so that $P=0$. Suppose by contradiction that $\gamma(0 \in \square)<\frac{q}{u_{d+1, q}}$. By the simplex trick, there exists a simplex $S$ with vertices among those of $\square$, which contains 0 in its relative interior, and $\gamma(0 \in \square) \geq \gamma(0 \in S)$. 
Let $\operatorname{dim} S=d^{\prime} \leq d$. Then $\gamma(0 \in S) \leq \gamma(0 \in \square)<\frac{q}{u_{d+1, q}} \leq \frac{q}{u_{d^{\prime}+1, q}}$. By Theorem 4.7, there exists $0 \neq e \in \mathbb{Z}^{d} \cap \operatorname{relint}\left(\frac{1}{q} S\right)$. Then $0, e, 2 e, \ldots, q e$ are $q+1$ distinct lattice points in the relative interior of $S$. They must be contained in $\mathbb{Z}^{d} \cap$ int $\square$. Contradiction!

As in [11, Theorem 3.6, Corollary 3.7], we obtain

Corollary 4.9. Let $\mathbb{Z}^{d}, \square, q$ as above. Then $\operatorname{vol}_{\mathbb{Z}^{d}}(\square) \leq q\left(\frac{u_{d+1, q}}{q}\right)^{d}$ and $\mathbb{Z}^{d} \cap \square$ has cardinality at most $d+d ! q\left(\frac{u_{d+1, q}}{q}\right)^{d}$.

Errata to [3]. The upper bound $n \leq c_{d} q^{d}$ in [3, Theorem 1.1] is not correct. In Step 1 of the proof, the constant $\gamma$ depends not only on $d-1$, but on $q$ as well. Since $\Lambda \simeq \mathbb{Z}^{d-1}$, S has vertices in $\frac{1}{q} \Lambda$ and $\Lambda \cap \operatorname{int}(S)=\{0\}$, Lemma 4.10 gives $\gamma \geq \frac{q}{u_{d, q}}$. Step 2 of the proof gives $j \leq d ! q^{d-1} \gamma^{-d+1}$. Since $j a \in \mathbb{Z}$, we obtain a correct effective upper bound for Theorem 1.1

$$
n \leq d ! u_{d, q}^{d-1} q .
$$

This bound is probably not sharp.

Lemma 4.10. Let $S$ be a simplex with vertices in $\frac{1}{q} \mathbb{Z}^{d}$, and such that $\{0\}=\mathbb{Z}^{d} \cap \operatorname{int}(S)$. Then $\gamma(0 \in S) \geq \frac{q}{u_{d+1, q}}$.

Proof. The simplex $S^{\prime}=q S$ has vertices in $\mathbb{Z}^{d}$ and $\{0\}=\mathbb{Z}^{d} \cap \operatorname{int}\left(\frac{1}{q} S^{\prime}\right)$. By Theorem $4.7, \gamma(0 \in$ $\left.S^{\prime}\right) \geq \frac{q}{u_{d+1, q}}$. But $\gamma\left(0 \in S^{\prime}\right)=\gamma(0 \in S)$.

\section{TORIC LOG FANO VARIETIES}

Let $(X, B)$ be a toric log variety, that is a log variety such that $X=T_{N} \operatorname{emb}(\Delta)$ is a toric variety and $B$ is an invariant $\mathbb{Q}$-Weil divisor. If $\sum_{i} E_{i}$ is the complement of the torus in $X$, we have $B=\sum_{i}\left(1-a_{i}\right) E_{i}$. Since $K_{X}+\sum_{i} E_{i}=0$, we obtain

$$
K_{X}+B=\sum_{i}-a_{i} E_{i}
$$

Let $\sigma \in \Delta(t o p)$. There exists $\psi_{\sigma} \in M_{\mathbb{Q}}$ such that $\left(\chi^{\psi_{\sigma}}\right)+K_{X}+B$ is zero on the open subset $U_{\sigma}$ of $X$. That is $\left\langle\psi_{\sigma}, e_{i}\right\rangle=a_{i}$ for every $e_{i} \in \sigma(1)$.

Minimal log discrepancies. Each $e \in N^{\text {prim }} \cap \sigma$ defines a toric valuation $E_{e}$ over $U_{\sigma}$, with log discrepancy computed by the formula

$$
a\left(E_{e} ; X, B\right)=\left\langle\psi_{\sigma}, e\right\rangle
$$

Since log resolutions exist in the toric category, we obtain

$$
\operatorname{mld}\left(U_{\sigma} ; X, B\right)=\min \left\{\left\langle\psi_{\sigma}, e\right\rangle ; 0 \neq e \in N \cap \sigma\right\} .
$$

The moment polytope associated to the $\mathbb{Q}$-divisor $-K_{X}-B$ is

$$
\square_{-K_{X}-B}=\left\{m \in M_{\mathbb{R}} ;\left\langle m, e_{i}\right\rangle+a_{i} \geq 0 \forall e_{i} \in \Delta(1)\right\} .
$$

It contains the origin of $M$. If $a_{i}>0$ for every $i$, denote by $P$ the convex hull of $\frac{e_{i}}{a_{i}}\left(e_{i} \in \Delta(1)\right)$. We compute $P^{*}=\square$. By duality,

$$
\inf \{t>0 ; e \in t P\}=-h_{\square}(e) \forall e \in N_{\mathbb{R}} .
$$


Toric weak $\log$ Fano varieties. Suppose moreover that $X$ is proper and $-K_{X}-B$ is $\mathbb{Q}$-semiample. Note that $-K_{X}-B$ is $\mathbb{Q}$-semiample if and only if it is nef. And if $a_{i}>0$ for every $i$, the $\mathbb{Q}$-divisor $-K_{X}-B$ is necessarily big.

The $\mathbb{Q}$-semiampleness condition is equivalent to $-\psi_{\sigma} \in \square$, for every $\sigma \in \Delta($ top $)$. And $-K_{X}-B$ is $\mathbb{Q}$-ample if and only if the vertices of $\square$ are precisely $\left(-\psi_{\sigma}\right)_{\sigma \in \Delta(t o p)}$. Let $e \in N^{\text {prim }}$, let $\sigma \in \Delta($ top $)$ contain $e$. Then $\square+\psi_{\sigma} \subseteq \sigma^{\vee}$. Therefore $\left\langle-\psi_{\sigma}, e\right\rangle=h_{\square}(e)$. Therefore

$$
a\left(E_{e} ; X, B\right)=-h_{\square}(e)=-\inf _{m \in \square}\langle m, e\rangle .
$$

We obtain the global formula $\operatorname{mld}(X, B)=-\sup _{0 \neq e \in N} h_{\square}(e)$. By duality,

$$
\operatorname{mld}(X, B)=\inf \{t>0 ;\{0\} \subsetneq N \cap t P\} .
$$

By Corollary 3.5, the $\alpha$-invariant of $-K_{X}-B$ with respect to $(X, B)$ is computed by the formula

$$
\gamma\left(X, B ;-K_{X}-B\right)=\gamma(0 \in \square)
$$

By duality, $\gamma(0 \in \square)=\gamma\left(0 \in \square^{*}\right)$. Denote $d=\operatorname{dim} X$.

Theorem 5.1. Let $q$ be a positive integer. If $\operatorname{mld}(X, B) \geq \frac{1}{q}$, then $\gamma(0 \in \square) \geq \frac{q}{u_{d+1, q}}$.

Proof. The assumption gives $a_{i} \geq \frac{1}{q}$ for every $i$. Suppose by contradiction that $\gamma<\frac{q}{u_{d+1, q}}$. By the simplex trick, there exists a simplex $S$, with vertices among those of $\square^{*}$, such that $0 \in$ relint $S$ and $\gamma(0 \in S) \leq \gamma\left(0 \in \square^{*}\right)$. Therefore

$$
\gamma(0 \in S)<\frac{q}{u_{d+1, q}} .
$$

Let $d^{\prime} \leq d$ be the dimension of $S$, and $\frac{e_{i}}{a_{i}}\left(0 \leq i \leq d^{\prime}\right)$ its vertices. Let $\gamma_{0}, \ldots, \gamma_{d^{\prime}}>0, \sum_{i=0}^{d^{\prime}} \gamma_{i}=1$ and $0=\sum_{i=0}^{d^{\prime}} \gamma_{i} \frac{e_{i}}{a_{i}}$. We have $\gamma(0 \in S)=\min _{i=0}^{d^{\prime}} \gamma_{i}$. Say $\gamma_{0}$ is minimal. Then $\gamma_{0}<\frac{q}{u_{d+1, q}} \leq \frac{q}{u_{d^{\prime}+1, q}}$, that is $\sum_{i=1}^{d^{\prime}} \gamma_{i}>\sum_{i=1}^{d^{\prime}} \frac{q}{u_{d^{\prime}+1, q}}$. Note that $1 \leq q a_{i} \leq q$. By Theorem 4.6, there exists $z \in \mathbb{N}^{d^{\prime}} \backslash 0$ such that

$$
\frac{q a_{i} z_{i}}{1+\sum_{j=1}^{d^{\prime}} q a_{j} z_{j}}<\gamma_{i}\left(1 \leq i \leq d^{\prime}\right) .
$$

These inequalities are equivalent to

$$
\frac{1}{q}>\max _{i=1}^{d^{\prime}} \frac{a_{i} z_{i}}{\gamma_{i}}-\sum_{j=1}^{d^{\prime}} a_{j} z_{j} .
$$

The right hand side is the smallest $t>0$ such that $e=\sum_{i=1}^{d^{\prime}}-z_{i} e_{i}$ belongs to $t S$. Therefore qe belongs to the relative interior of $S$, so in the relative interior of $P$ as well.

Let $e^{\prime}=e / n$ be the primitive vector on the ray generated by $e$. Then $e^{\prime}$ defines a toric valuation of $X$ with $\log$ discrepancy $a\left(E_{e^{\prime}} ; X, B\right)<\frac{1}{q n}$. Therefore $\operatorname{mld}(X, B)<\frac{1}{q}$, a contradiction.

Corollary 5.2. If $\operatorname{mld}(X, B) \geq \frac{1}{q}$, then $\sqrt[d]{\left(-K_{X}-B\right)^{d}} \leq \frac{d}{q} u_{d+1, q}$.

Proof. By Proposition $2.10, \gamma \cdot \sqrt[d]{\left(-K_{X}-B\right)^{d}} \leq d$.

Example 5.3. The lower bound in Theorem 5.1 is sharp. In dimension one, $\left(\mathbb{P}^{1}, \frac{q-1}{q} \cdot \infty\right)$ is the only example which attains it. A higher dimensional example is constructed as follows. Let $e_{1}, \ldots, e_{d}$ be the standard basis of $\mathbb{Z}^{d}$, and

$$
e_{0}=\sum_{i=1}^{d}-\frac{u_{d+1, q}}{q\left(1+u_{i, q}\right)} e_{i}
$$


Then $e_{0}, \ldots, e_{d}$ are primitive vectors in the lattice $N=\mathbb{Z}^{d}$, which they generate. Set $a_{0}=\frac{1}{q}$ and $a_{1}=\cdots=a_{d}=1$. This defines a toric $\log$ Fano $\left(X^{d},\left(1-\frac{1}{q}\right) E_{0}\right)$ with

$$
\begin{aligned}
\square_{-K_{X}-B} & =\left\{m \in \mathbb{R}_{\geq-1}^{d} ; \sum_{i=1}^{d} \frac{u_{d+1, q}}{1+u_{i, q}} m_{i} \leq 1\right\} \\
& =(-1, \ldots,-1)+\left\{m \in \mathbb{R}_{\geq 0}^{d} ; \sum_{i=1}^{d} \frac{1}{1+u_{i, q}} m_{i} \leq \frac{1}{q}\right\}
\end{aligned}
$$

The simplex $\square_{-K_{X}-B}=\operatorname{Conv}\left(v_{0}, \ldots, v_{d}\right)$ contains in interior the origin of $M$, with barycentric coordinates

$$
0=\frac{q}{u_{d+1, q}} v_{0}+\sum_{i=1}^{d} \frac{q}{1+u_{i, q}} v_{i}
$$

So $\gamma\left(X, B ;-K_{X}-B\right)=\frac{q}{u_{d+1, q}}$. One checks that $\operatorname{mld}(X, B)=\frac{1}{q}$, Bs $|-q(K+B)|=\emptyset$ and $\left(-q\left(K_{X}+B\right)\right)^{d}=\frac{u_{d+1, q}}{q}$. In particular, the upper bound in Corollary 5.2 is not sharp.

Theorem 5.4 (cf. [7]). Fix $d \geq 1$ and $\epsilon \in(0,1]$. Consider toric proper varieties $X$ such that $\operatorname{dim} X=d$ and there exists an invariant effective $\mathbb{Q}$-divisor $B$ such that $-K_{X}-B$ is $\mathbb{Q}$-semiample and $\operatorname{mld}(X, B) \geq \epsilon$. Then $X$ belongs to finitely many isomorphism types.

Proof. Let $\gamma=\gamma\left(0 \in \square_{-K_{X}-B}\right)=\gamma\left(0 \in P_{-K_{X}-B}\right)$. By Theorem [5.1, $\gamma \geq \gamma(d, \epsilon)>0$. We have $\epsilon \gamma(P-P) \subseteq \epsilon P$. Therefore $\{0\}=N \cap \operatorname{int}(\epsilon \gamma(P-P))$. Theorem 4.1 gives

$$
\operatorname{vol}_{N}(P-P) \leq \frac{1}{\gamma^{d} \epsilon^{d}}
$$

Let $C$ be the convex hull of $\Delta(1)$. Since $C \subseteq P$, we deduce that $\operatorname{vol}_{N}(C-C)$ is bounded above. Since $C$ is a lattice polytope, it follows that the pair $(N, C-C)$ belongs to finitely many isomorphism types. There exist only finitely many fans $\Delta$ with given $\Delta(1)$. Therefore $(N, \Delta)$ belongs to finitely many isomorphism types.

Examples. Consider toric log Fano varieties $(X, B)$, of Picard number one, and such that $X$ has no nontrivial toric finite covers which are étale in codimension one. Let $X=T_{N} \operatorname{emb}(\Delta), \operatorname{dim} N=d$. Then $\Delta(1)=\left\{e_{0}, \ldots, e_{d}\right\}$, where $e_{0}, \ldots, e_{d} \in N$ are primitive and generate the lattice $N$, and no $d$ of them are linearly dependent. The log discrepancies in invariant prime divisors are rational numbers $a_{0}, \ldots, a_{d} \in[0,1]$, not all zero. We have

$$
0=\sum_{i=0}^{d} x_{i} e_{i}\left(x_{i} \in \mathbb{Q}_{>0}, \sum_{i} x_{i}=1\right) .
$$

The vector $e_{i}$ is primitive in the lattice generated by $e_{0}, \ldots, e_{d}$ if and only if $n_{i}=1$, where

$$
n_{i}=\frac{\operatorname{gcd}\left(q x_{0}, \ldots, \widehat{q x_{i}}, \ldots, q x_{d}\right)}{\operatorname{gcd}\left(q x_{0}, \ldots, q x_{d}\right)}\left(q \geq 1, q x \in \mathbb{Z}^{d+1}\right) .
$$

Up to isomorphism, $(X, B)$ is uniquely determined by $\left(x_{i}\right)_{i}$ and $\left(a_{i}\right)_{i}$. The dual polytope $\square_{-K_{X}-B}^{*}$ is the simplex with vertices $e_{i} / a_{i}$.

The dual description of $(X, B)$ is: $0 \in \square \subset M_{\mathbb{R}}$ is a rational simplex with vertices $v_{0}, \ldots, v_{d}$, and 0 lies at distance $a_{i}$ to the face of $\square$ opposite $v_{i}$ (distance measured with respect to $e_{i}$, the primitive interior direction normal to the face opposite to $v_{i}$ ). Let

$$
0=\sum_{i=0}^{d} \gamma_{i} v_{i}\left(\gamma_{i} \geq 0, \sum_{i} \gamma_{i}=1\right)
$$


Then $\gamma_{i}=\frac{a_{i}}{w_{i}}$, where $w_{i}=\operatorname{width}\left(\square ; e_{i}\right)$. The above data are related as follows:

- $\left\langle v_{i}, e_{j}\right\rangle+a_{j}=0$ for $i \neq j$, and $\left\langle v_{j}, e_{j}\right\rangle+a_{j}=w_{j}$.

- $\left(\sum_{i} a_{i} x_{i}\right)\left(\sum_{i} \frac{1}{w_{i}}\right)=1$.

- $x_{j}=\frac{\frac{1}{w_{j}}}{\sum_{i=0}^{d} \frac{1}{w_{i}}}, \gamma_{j}=\frac{a_{j} x_{j}}{\sum_{i=0}^{d} a_{i} x_{i}}, w_{j}=\frac{\sum_{i} a_{i} x_{i}}{x_{j}}$.

We have $K_{X}+B+\sum_{i=0}^{d} a_{i} E_{i} \sim 0$. For $0 \leq i \leq d$, set $D_{i}=\left(\chi^{v_{i}}\right)-K_{X}-B=w_{i} E_{i}$. So $K_{X}+B+D_{i} \sim_{\mathbb{Q}} 0$ and $\operatorname{lct}\left(X, B ; D_{i}\right)=\gamma_{i}$.

Note that $B=0$ if and only if $a_{i}=1$ for all $i$, and then $x_{i}=\gamma_{i}=\frac{1}{w_{i}}$ for all $i$.

Lemma 5.5. $r\left(K_{X}+B\right)$ is Cartier if and only if $\mathrm{Bs}\left|-r\left(K_{X}+B\right)\right|=\emptyset$, if and only if $r a_{i}, r w_{i} \in \mathbb{Z}$ for every $i$.

Lemma 5.6. $\gamma\left(X, B ;-K_{X}-B\right)=\min _{i=0}^{d} \gamma_{i}$.

Lemma 5.7. Let $0<\epsilon \leq \min _{i=0}^{d} a_{i}$. Then $\operatorname{mld}(X, B) \geq \epsilon$ if and only if there does not exist an integer $n \geq 0$ such that $\sum_{i}\left\{(n+1) x_{i}\right\}=1,\{(n+1) x\} \neq x$, and

$$
\sum_{i=0}^{d} a_{i}\left\{(n+1) x_{i}\right\}-\epsilon<\min _{j=0}^{d} w_{j}\left\{(n+1) x_{j}\right\} .
$$

The fractional parts of vectors are defined componentwise.

Proof. We clearly have $\operatorname{mld}(X, B)=a \leq \min _{i=0}^{d} a_{i}$. Log discrepancies in toric valuations are computed as follows: if $e=\sum_{i=0}^{d} t_{i} e_{i}$ is a primitive vector in $N$, the induced toric valuation $E_{e}$ has $\log$ discrepancy

$$
a\left(E_{e} ; X, B\right)=\inf \left\{\epsilon>0 ; \sum_{i=0}^{d} t_{i} e_{i} \in \epsilon \cdot \square^{*}\right\}=\max _{j=0}^{d} \sum_{i=0}^{d} a_{i} t_{i}-w_{j} t_{j} .
$$

We have $a \cdot \square^{*} \subseteq C=\operatorname{Conv}\left(e_{0}, \ldots, e_{d}\right)$. Therefore the primitive vectors which attain $a$ must be contained in $C$. But $N \cap C$ is parametrized as follows:

$$
N \cap C \backslash\left\{e_{i} ; i\right\}=\left\{\sum_{i}\left\{(n+1) x_{i}\right\} e_{i}=\sum_{i}-\left\lfloor(n+1) x_{i}\right\rfloor e_{i} ; n \geq 0, \sum_{i}\left\{(n+1) x_{i}\right\}=1\right\} .
$$

Note $\sum_{i}\left\{(n+1) x_{i}\right\}=1$ if and only if $\sum\left\lfloor(n+1) x_{i}\right\rfloor=n$. And $\sum_{i}\left\{(n+1) x_{i}\right\} e_{i}=0$ if and only if $\{(n+1) x\} \neq x$, if and only if $n x \in \mathbb{Z}^{d+1}$. We obtain

$$
a=\min \left(\min _{i=0}^{d} a_{i}, \min _{\sum_{i}\left\{(n+1) x_{i}\right\}=1,\{(n+1) x\} \neq x} \sum_{i} a_{i}\left\{(n+1) x_{i}\right\}-\min _{j} w_{j}\left\{(n+1) x_{j}\right\}\right)
$$

Lemma 5.7 has a geometric reformulation: let $S=\left\{x \in \mathbb{R}_{\geq 0}^{d+1} ; \sum_{i=0}^{d} x_{i}=1\right\}$, with vertices $P_{0}, \ldots, P_{d}$. Our point $x=\sum_{i} x_{i} P_{i}$ lies in the interior of $S$. Let $S_{\epsilon}(x)$ be the simplex with vertices $Q_{i}=\left(1-\frac{\epsilon}{a_{i}}\right) x+\frac{\epsilon}{a_{i}} P_{i}(0 \leq i \leq d)$. It is a neighborhood of $x$ in $S$ obtained by sliding each vertex of $S$ towards $x$, with a certain weight. Then:

- $\operatorname{mld}(X, B) \geq \epsilon$ if and only if int $S_{\epsilon}(x)$ contains no $\{(n+1) x\}$ other than $x$.

- $x=\sum_{i} \gamma_{i} Q_{i}$.

So both $\operatorname{mld}(X, B)$ and $\gamma\left(X, B ;-K_{X}-B\right)$ are encoded by the neighborhood $x \in S_{\epsilon}(x)$. The condition $\operatorname{mld}(X, B) \geq \epsilon$ means that the rational point $x$ is badly approximated by the fractional parts of its multiples, and Theorem 5.4 states that such $x$ belong to a finite set.

Lemma 5.8. Let $\epsilon \leq \min _{i=0}^{d} a_{i}$. Then $\operatorname{mld}(X, B)<\epsilon$ if and only if one of the following equivalent conditions hold: 
a) There exists $n \geq 1$ such that $\sum_{i=0}^{d}\left\{(n+1) x_{i}\right\}=1,\{(n+1) x\} \neq x$, and

$$
\frac{\sum_{i=0}^{d} a_{i}\left\{(n+1) x_{i}\right\}-\epsilon}{\sum_{i=0}^{d} a_{i} x_{i}}<\min _{j=0}^{d} \frac{\left\{(n+1) x_{j}\right\}}{x_{j}} \text {. }
$$

b) There exists $z \in \mathbb{N}^{d+1}$ such that $\max _{j} w_{j} z_{j}-\sum_{i} a_{i} z_{i} \in(0, \epsilon)$.

Moreover, $z=\lfloor(1+|z|) x\rfloor$. So if $z$ exists, it is uniquely determined by $|z|$ and $x$. If $\left.q x \in \mathbb{Z}^{d+1}, b\right)$ can be decided by considering only the multiples $x, 2 x, \ldots,(q-1) x$.

Example 5.9. Suppose $B=0$. That is $a_{i}=1, x_{i}=\gamma_{i}=\frac{1}{w_{i}}$. Then $\operatorname{mld}(X, 0)<\epsilon$ if and only if there exists $n \geq 1$ such that $\{(n+1) x\} \neq x$ and $1-\epsilon<\min _{j} \frac{\left\{(n+1) x_{j}\right\}}{x_{j}}$, if and only if there exists $z \in \mathbb{N}^{d+1} \backslash 0$ such that $\frac{z_{j}}{\epsilon+\sum_{i=0}^{d} z_{i}}<x_{j}$ for every $j$.

\section{REFERENCES}

[1] Alexeev, V., Boundedness and $K^{2}$ for log surfaces. Internat. J. Math. 5(6) (1994), $779-810$.

[2] Ambro, F., The minimal log discrepancy, preprint math/0611859 (2006).

[3] Ambro, F., On the classification of toric singularities, in Proceedings of the Conference on Combinatorial Commutative Algebra and Computer Algebra (Mangalia 2008), V. Ene and E. Miller (Ed.), Contemporary Mathematics 502 (2009), 1-4

[4] Ambro, F., Basic properties of log canonical centers, in Classification of Algebraic Varieties, C. Faber, G. van der Geer, E. Looijenga (Ed.), EMS Series of Congress Reports, 2011, vol. 3, 39 - 48

[5] Averkov, G., On the size of lattice simplices with a single interior lattice point. SIAM J. Discrete Math. 26 (2012), no. 2, 515-526.

[6] Averkov, G.; Krümpelmann J.; Nill B., Largest integral simplices with one interior integral point: solution of Hensley's conjecture and related results. preprint arXiv: 1309.7967.

[7] Borisov, A. A.; Borisov, L. A., Singular toric Fano varieties. (Russian) Mat. Sb. 183(2) (1992), 134-141; translation in Russian Acad. Sci. Sb. Math. 75(1) (1993), 277-283.

[8] Delcroix, T., Alpha-invariant of Toric Line Bundles. preprint arXiv:1409.0961 (2014).

[9] Demailly, J.-P.; Kollár, J., Semi-continuity of complex singularity exponents and KählerEinstein metrics on Fano orbifolds. Annales scientifiques de l'École Normale Supérieure 34(4)(2001), 525 - 556.

[10] Hassett, B., Kovács, S.J., Reflexive pull-backs and base extension. J. Algebraic Geometry 13 (2004), 233247.

[11] Hensley, D., Lattice vertex polytopes with interior lattice points. Pacific J. Math. 105(1) (1983), 183-191.

[12] Kawakita, M., Inversion of adjunction on log canonicity. Invent. Math. 167 (2007), no. 1, 129 - 133.

[13] Kollár, J., Singularities of pairs, Algebraic Geometry, Santa Cruz, 1995; Proceedings of Symposia in Pure Math. vol. 62, AMS (1997), $221-287$.

[14] Lekkerkerker, C. G., Geometry of numbers. Bibliotheca Mathematica, Vol. VIII Wolters-Noordhoff Publishing, Groningen; North-Holland Publishing Co., Amsterdam-London 1969.

[15] Soundararajan, K., Approximating 1 from below using $n$ Egyptian fractions. preprint arXiv:math/0502247 [math.CA].

[16] Varchenko A.N., Complex exponents of a singularity do not change along the stratum $\mu=$ constant, Functional Anal. Appl.16 (1982) 1 - 9.

[17] Viehweg, E., Quasi-Projective Moduli of Polarized Manifolds, Springer-Verlag, Berlin, 1995.

Institute of Mathematics "Simion Stollow" of the Romanian Academy, P.O. BOX 1-764, RO014700 Bucharest, Romania.

E-mail address: florin.ambro@imar.ro 